\title{
Evolution of gas disc-embedded intermediate mass ratio inspirals in the LISA band
}

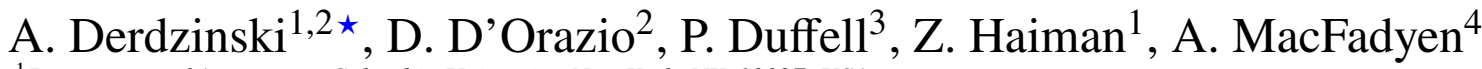 \\ ${ }^{1}$ Department of Astronomy, Columbia University, New York, NY, 10027, USA \\ ${ }^{2}$ Center for Theoretical Astrophysics and Cosmology, Institute for Computational Science, University of Zurich, \\ Winterthurerstrasse 190, 8057 Zurich, Switzerland \\ ${ }^{3}$ Department of Astronomy, Harvard University, 60 Garden Street Cambridge, MA 01238, USA \\ ${ }^{4}$ Center for Cosmology and Particle Physics, Physics Department, New York University, New York, NY 10003,USA
}

Received / Accepted

\begin{abstract}
Among the potential milliHz gravitational wave (GW) sources for the upcoming space-based interferometer LISA are extreme- or intermediate-mass ratio inspirals (EMRI/IMRIs). These events involve the coalescence of supermassive black holes in the mass range $10^{5} M_{\odot} \lesssim M \lesssim$ $10^{7} M_{\odot}$ with companion BHs of much lower masses. A subset of E/IMRIs are expected to occur in the accretion discs of active galactic nuclei (AGN), where torques exerted by the disc can interfere with the inspiral and cause a phase shift in the GW waveform. Here we use a suite of two-dimensional hydrodynamical simulations with the moving-mesh code DISCO to present a systematic study of disc torques. We measure torques on an inspiraling BH and compute the corresponding waveform deviations as a function of the binary mass ratio $q \equiv M_{2} / M_{1}$, the disc viscosity $(\alpha$ ), and gas temperature (or equivalently Mach number; $\mathcal{M}$ ). We find that the absolute value of the gas torques is within an order of magnitude of previously determined planetary migration torques, but their precise value and sign depends non-trivially on the combination of these parameters. The gas imprint is detectable by LISA for binaries embedded in AGN discs with surface densities above $\Sigma_{0} \geq 10^{4-6} \mathrm{gcm}^{-2}$, depending on $q, \alpha$ and $\mathcal{M}$. Deviations are most pronounced in discs with higher viscosities, and for E/IMRIs detected at frequencies where LISA is most sensitive. Torques in colder discs exhibit a noticeable dependence on the GW-driven inspiral rate as well as strong fluctuations at late stages of the inspiral. Our results further suggest that LISA may be able to place constraints on AGN disc parameters and the physics of disc-satellite interaction.
\end{abstract}

Key words: black hole physics, gravitational waves, hydrodynamics

\section{INTRODUCTION}

In the 2030s we expect to detect binary mergers involving massive black holes (MBHs; in the mass range $M_{\mathrm{BH}} \sim 10^{4}-10^{7} M_{\odot}$ ) across the universe with the space-based gravitational wave $(\mathrm{GW})$ detector LISA (Amaro-Seoane et al. 2017). LISA will also detect extreme and intermediate mass ratio mergers (termed EMRIs, $q \equiv M_{2} / M_{1} \lesssim$ $10^{-4}$; or IMRIs, $q \approx 10^{-3}-10^{-4}$ ) up to a redshift $z \sim 4$.

Many near-equal mass MBH mergers in the LISA band may occur in gaseous environments, given that the galactic mergers that lead to the eventual formation of a MBH binary often carry a fresh supply of gas into the post-merger galactic nucleus (see Mayer 2013 for a review). Likewise, "gas-embedded" E/IMRIs may frequently occur in the accretion discs expected in active galactic nuclei (AGN) in which an $\mathrm{MBH}$ is surrounded by a thin, dense accretion disc. Compact objects in the nucleus can eventually align their orbits with

^ E-mail: andrea@ics.uzh.ch the disc, provided a sufficient number of disc-crossing intersections (e.g. Syer et al. 1991; Artymowicz et al. 1993; Rauch 1995; Just et al. 2012; McKernan et al. 2012; Kennedy et al. 2016; Artymowicz et al. 1993; Fabj et al. 2020; Panamarev et al. 2018; MacLeod \& Lin 2020), or such events may arise from in-situ star formation in the disc that leaves compact remnants (Goodman \& Tan 2004; Levin 2007). Embedded stars and BHs can subsequently accrete, migrate, and merge with each other (Bellovary et al. 2016; Tagawa et al. 2020a; Secunda et al. 2019, 2020), before eventually merging with the central MBH. While the event rate for E/IMRIs has previously been estimated in dry nuclei (e.g. Barausse et al. 2015), the rate may be considerably higher when including formation pathways in accretion discs. The recent discovery of stellar-mass BH mergers by the ground based interferometer LIGO has stimulated work on such mergers that may be occurring in AGN discs, showing that they might contribute to LIGO events (McKernan et al. 2014; Bartos et al. 2017; McKernan et al. 2018; Gröbner et al. 2020; Stone et al. 2017). Subsequent work has suggested that some of the LIGO events, based 
on their high chirp masses, may indeed have formed via this channel - large masses are expected both because of the preferential capture of heavier BHs by the disc (Yang et al. 2019b) and because repeated mergers are common and lead to hierarchical build-up (Yang et al. 2019a). The high effective spin provides additional support for this channel (Gayathri et al. 2020) as well as events with more unequal mass ratios (e.g. GW190412, see Tagawa et al. 2020b) which have been predicted to occur naturally via dynamical interactions in a dense, gaseous environment (McKernan et al. 2020; Tagawa et al. 2020a; Secunda et al. 2020). These results suggest that BH mergers indeed occur in gas discs, and that they could constitute the building blocks of IMBHs (McKernan et al. 2012; Tagawa et al. 2020a). Recent semi-analytical estimates of in-situ star formation suggest that the accretion of embedded compact objects may also contribute substantially to the growth of MBHs (Dittmann \& Miller 2020).

In general, whenever gas is present around a coalescing MBH binary, the torques exerted by the gas can influence the inspiral rate. For a near-equal mass binary in the late inspiral stage, these torques are noticeable only at extreme gas densities, comparable to those expected if the binary is embedded in a common envelope phase (Antoni et al. 2019; Chen \& Shen 2019). For extreme- and intermediate- mass ratio systems, the GW torques are weaker and gas torques on the lower-mass companion are stronger. As a result, for a long-lived E/IMRI detected and monitored by several years with the low-frequency GW detector LISA, gas torques can become more comparable to (albeit still well below) GW torques, and impart a detectable imprint on the GW waveform (Yunes et al. 2011; Kocsis et al. 2011; Derdzinski et al. 2019). This presents a novel and unique opportunity for LISA to detect environmental influence in a MBH waveform and probe accretion disc physics.

A longstanding hurdle in numerically evaluating this effect is that gas torques are subtle, remain poorly understood, and show a complex dependence on system parameters (see $\S 2$ below). The response of a disc to an embedded satellite can be highly nonlinear (Baruteau \& Masset 2013), making simulations necessary to quantify it, and the resulting torque can be remarkably sensitive to small changes in the satellite mass or disc parameters (Duffell 2015). Solving for the disc torques requires especially careful considerations of numerical resolution, boundary effects and transients (i.e. achieving steady-state behaviour), as well as the accretion of both mass and momentum by the migrating BH (Tang et al. 2017; Muñoz et al. 2019; Moody et al. 2019; Muñoz et al. 2020). For a large range of mass ratios, simulations show that disc torques may either help or hinder the binary merger (Duffell et al. 2020).

In a previous paper (Derdzinski et al. 2019; hereafter Paper I), we simulated the gas response to an embedded IMRI (with mass ratio $q=10^{-3}$ ) in order to measure its detectability in the $\mathrm{GW}$ signal. We found that the disc torques slow down, rather than speed up the inspiral, due to a critical contribution to the torque that comes from the asymmetry in the gas morphology near the migrator (near or within the BH's Hill sphere). We estimated that the resulting deviation in the GW waveform, which comprises of a slow drift in the accumulated GW phase, is detectable if the IMRI resides in a disc with surface densities above $\Sigma_{0} \gtrsim 10^{3-4} \mathrm{~g} \mathrm{~cm}^{-2}$.

In Paper I, we considered only a single set of binary system and disc parameters. In order to extract meaningful information from environmental imprints on a GW waveform, we must understand the range of possible effects. In particular, we need to know how the torques and the corresponding waveform deviations depend on system parameters (mass ratio, eccentricity, inclination, spins) and the source environment (in our case, properties of the disc such as density, temperature or viscosity). In the present paper, we further explore the scaling of the torque with a subset of these parameters in the regime where GW-emission is dominant.

This work is motivated by the idea that, provided we understand how gas torques impact a GW inspiral, GWs can be used as a tool for providing measurements of AGN disc properties, and to improve our understanding of the physics of migration. To this end, we study disc torques as a function of the GW inspiral rate in order to isolate the effect of the inspiral, and to assess how the disc torques may evolve differently for different combinations of parameters. We restrict this study to include only three parameters - namely the binary mass ratio $(q)$ and two of the disc parameters: temperature (or equivalently Mach number; $\mathcal{M}$ ) and viscosity (parameterised by the Shakura-Sunyaev parameter $\alpha$ ). As in Paper I, we measure the torques directly in hydrodynamical simulations over a limited range of binary separations, and then extrapolate these measurements to cover the final coalescence of a physical IMRI, covering several years of LISA observations. Scaling our simulations to physical parameters, we calculate the detectability of the gas imprint on the GW signal across our set of simulated mass ratios, and place constraints on the minimum AGN disc density required to detect the gas-induced deviations on the GW waveform. We show that the detectability of these deviations depends on the mass ratio and also the stage at which the inspiral is observed; we also find that the time-evolution of the gas torques in the LISA window depends non-trivially on $q, \mathcal{M}$, and $\alpha$.

This paper is organised as follows. In $\S 2$, we begin by summarising prior work. In $\$ 3$, we describe our numerical approach, including the hydrodynamical simulations and the range of simulated parameters. In $\S 4$, we present our results, measuring and analysing the gas torques and their dependence on each parameter. In $\S 5$, we apply our results to LISA binaries, calculating the detectability of the gas-induced deviations in the waveform. Finally, we discuss our results in $\S 6$, and summarize our conclusions in $\S 7$.

\section{PREVIOUS WORK}

Here we summarize (i) applicable work on planetary torques for intermediate mass ratio systems, (ii) analytical work on disc torques on GW inspirals, and (iii) our previous paper that combines the two with simulations.

To understand the evolution of a low-mass satellite embedded in a gas disc, the large majority of the work to date has been done in the context of planetary migration in protoplanetary discs. The embedded satellite perturbs the disc non-axisymmetrically, and these perturbations back-react on the satellite's orbit. The perturbations include spiral density waves and gaps where streams of gas can flow on horseshoe orbits around the satellite (see e.g. Baruteau \& Masset 2013 for a comprehensive review). Historically, two distinct regimes have been identified and known as Type I and Type II migration (Ward 1997), determined by the mass of the embedded satellite, as well as by the disc temperature and viscosity. In the Type I regime (for mass ratios $q \lesssim 10^{-4}$, for typical disc temperatures and viscosities), the disc response is linear, and the migration rate can be predicted analytically (Goldreich \& Tremaine 1980) and described with simple formulae (such as in Tanaka et al. 2002) that have been confirmed and calibrated with two- and three-dimensional simulations (e.g., Dong et al. 2011; D’Angelo \& Lubow 2010; Duffell \& MacFadyen 2012) although these predictions assume that the disc is locally isothermal (Paardekooper \& Mellema 2006). In the Type II regime (or gap-opening regime) (typically $q \gtrsim 10^{-4}$ ), the disc response becomes nonlinear and the secondary begins to carve 
a low-density annular gap. The migration rate typically scales with some fraction of the local viscous rate (e.g. Edgar 2007) and is proportional to the gas density in the gap (Kanagawa et al. 2018). However, the migration rate depends on disc parameters, and can even switch sign for certain combinations (Duffell et al. 2014; Duffell 2015). One distinct regime is when the local disc mass is much smaller than the satellite's mass. Disc torques in this regime are lowered significantly, causing migration to slow down (Syer \& Clarke 1995; Duffell et al. 2014). Note that the E/IMRIs considered in our study are in this regime. In summary, in both the Type I and Type II regimes, the disc response, especially in the co-orbital region of the satellite, and the resulting torques on the satellite, are sensitive to disc parameters and to the equation of state. This makes predictions for real systems (whose parameters are unknown) difficult to make (Kley \& Nelson 2012).

Despite these caveats, analytical predictions are convenient and are often utilised in the literature. In the context of BHs embedded in gas discs, prior work has estimated the impact of gas torques on LISA sources in order to assess their detectability in the GW waveform (Yunes et al. 2011; Kocsis et al. 2011; Barausse et al. 2014). These analytic studies adopted the gas torques as in the planet literature, and added these linearly to the effective GW torques. They have concluded that gas torques on EMRIs, which can spend up to $O\left(10^{5}\right)$ cycles in the LISA band, are potentially detectable if embedded in thin, near-Eddington accretion discs (i.e. discs whose steady-state accretion rate corresponds to the Eddington luminosity of the central SMBH, at a radiative efficiency of $\sim 10 \%$ ).

In Paper I, we improved on these analytic estimates by performing the first direct measurement of gas disc torques on a gasembedded IMRI, using two-dimensional hydrodynamical simulations. Unlike in planetary migration studies, the satellite was assumed to follow a GW-driven inspiral, and, unlike in the analytic estimates, we did not assume that that GW and disc torques can be added linearly. Contrary to analytical estimates, we found that a disc-embedded IMRI experiences outward gas torques that hinder the inspiral. This was shown for a single set of system and disc parameters: a binary mass ratio $q=10^{-3}$, viscosity parameter $\alpha=0.03$ and Mach number $\mathcal{M}=20$ (see below for the definitions of $\alpha$ and $\mathcal{M}$ ).

Paper I was the first to address how these torques may evolve during a GW-driven inspiral and to calculate their detectability directly from simulations. This demonstrated a proof-of-concept for an optimal case: a $10^{-3}$ mass-ratio inspiral is relatively loud (compared to EMRIs), and chirps substantially as it approaches merger. These two qualities are paramount to detect the corresponding waveform deviations, and to use the frequency-dependence of these deviations to distinguish them from variations in system parameters in order to securely identify them as environmental effects.

In the present work, we follow up on Paper I and explore how the detectability of disc torques in GW waveforms depends on system or disc parameters. Changing the mass ratio of the system will affect the gas dynamics (and the torque experienced by the secondary) as well as the GW evolution of the binary in the LISA band (and resulting detectability of the gas imprint). We expand on the IMRI parameter space over an order of magnitude of mass ratio, demonstrating that reducing the secondary mass leads to different outcomes in torque evolution and correspondingly more stringent constraints on detectability. We also then explore how these results depend on disc viscosity and Mach number. With a range of simulations we show that whether or not torques accelerate or hinder the GW-driven inspiral, and whether the resulting gas imprint is detectable in the GW data stream, will depend on the combinations of these parameters.

\section{SIMULATION SETUP}

We use the moving-mesh hydrodynamics grid code DISCO (Duffell 2016) to model a thin two-dimensional, viscous disc around an MBH with a low-mass satellite BH embedded in the disc, following a GWdriven inward migration ("inspiral"). In this section, we describe our scale-free numerical approach for the disc, the prescribed orbit of the migrator, as well as how we measure the torques.

\subsection{Disc model}

The simulation setup is the same as in Paper I, with slight modifications to the domain size and spatial resolution. We model a vertically-integrated, near-Keplerian disc, parameterised by a constant aspect ratio $h / r \equiv \mathcal{M}^{-1}$, where $h$ is the disc scale height, $r$ is the distance to the central MBH held at the origin, and $\mathcal{M}$ is the Mach number for the azimuthal velocity $v_{\phi}=\sqrt{G M_{1} / r}$ around the central MBH of mass $M_{1}$. For thin and cold discs, the latter is equivalent to specifying the local sound speed; $c_{s}=v_{\phi} / \mathcal{M}$. We adopt a constant $\alpha$-law prescription for the viscosity, such that the kinematic viscosity is set by $v(r)=\alpha c_{s}(r) h(r)$. We force the disc to be locally isothermal by setting the pressure to $p=c_{s} \Sigma(r)$, where $\Sigma(r)$ is the surface density (i.e. the vertically integrated density) at radius $r$. With the above constraints, the initial condition for the surface density profile becomes:

$\Sigma(r)=\Sigma_{0}\left(\frac{r}{r_{0}}\right)^{-1 / 2}$,

where $r_{0}$ is an arbitrary distance unit, and $\Sigma_{0}$ is a corresponding normalisation.

The simulation domain extends from $0.5<r / r_{0}<6.0$, with 666 logarithmically-spaced radial cells and an increasing number of azimuthal cells with radius in order to maintain a uniform cell aspect ratio. The disc is resolved at all radii to $\Delta r / r_{0} \lesssim 0.003$. Fixing the number of cells for all runs means that the resolution in terms of the disc scale height changes with Mach number, such that $\Delta r / h=\lesssim 0.04,0.07,0.1$ for $\mathcal{M}=10,20,30$, respectively. We performed a resolution test with 800 radial cells for our fiducial runs below, and confirmed that the torques did not change at this somewhat higher resolution. Compared to Paper I, we more than double the outer boundary of the simulation. We found that for more massive satellites, a closer-in outer boundary leads to numerical transients in the torque that last for longer than a viscous time.

To visualise our simulation, we show a snapshot of the 2D surface density distribution in Fig. 1.

\subsection{The inspiralling $\mathrm{BH}$}

The primary SMBH is excised from the simulation domain, and the secondary $\mathrm{BH}$ is modeled as a point mass with a smoothed gravitational potential, defined by

$\Phi_{2}=\frac{G M_{2}}{\left(r_{2}^{2}+\epsilon^{2}\right)^{1 / 2}}$,

where $G$ is the gravitational constant, $r_{2}$ is the distance to the secondary $\mathrm{BH}$ and $\epsilon=h(r) / 2$ is the smoothing length at the secondary's position. Note that $\epsilon$ is introduced here, as in other similar 


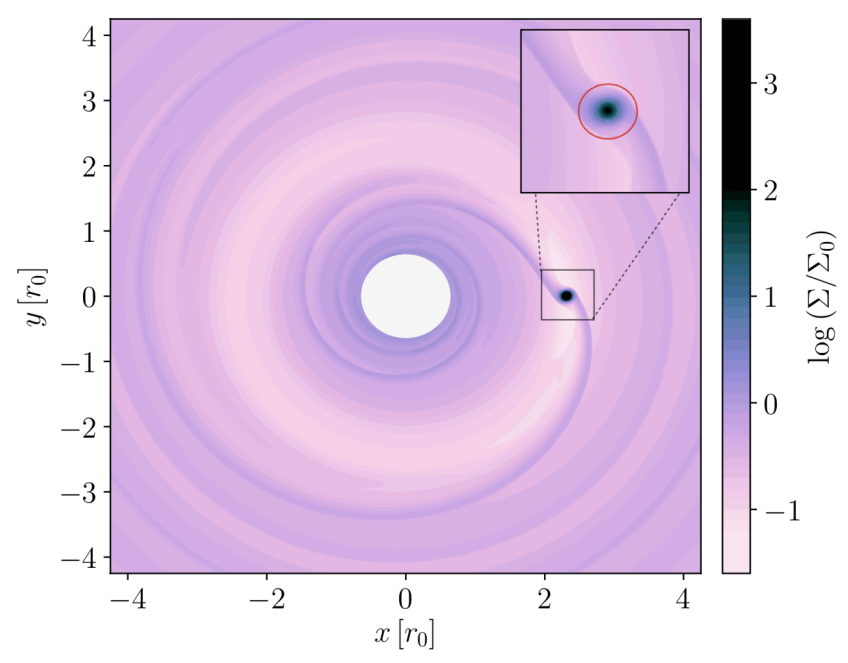

Figure 1. Snapshot of the surface density in a simulation with $q=10^{-3}$, $\alpha=0.03$, and $\mathcal{M}=20$ after 2500 orbits of the satellite. An embedded migrating perturber orbits counter-clockwise, excites spiral density waves, and carves a shallow gap. The zoom-in panel in the upper right corner shows a close-up with the color scaling altered to highlight streams flowing across the secondary's Hill radius (marked by the red circle).

works, to mimic the vertically integrated forces within a scale height of the $\mathrm{BH}$, and is continuously updated as the $\mathrm{BH}$ spirals inward.

Under the quadrupole approximation for gravitational radiation, for a circular GW inspiral (Peters 1964), the evolution of the separation of a binary driven together by $\mathrm{GW}$ emission follows

$\dot{r}_{\mathrm{GW}}=-\frac{64}{5} \frac{G^{3}}{c^{5}} \frac{M^{3}}{(1+1 / q)(1+q)} \frac{1}{r^{3}}$,

where $M=M_{1}+M_{2}$ is the total mass of the binary. Following this, the secondary $\mathrm{BH}$ is placed in a quasicircular, prograde orbit whose separation evolves according to

$r(t)=r_{\mathrm{f}}\left(1-4 R_{\mathrm{f}}\left(t-t_{\mathrm{tot}}\right)\right)^{1 / 4}$,

where $t$ is the elapsed time, $t_{\text {tot }}$ is the total simulation time, $r_{\mathrm{f}}$ is the final binary separation, and $R_{\mathrm{f}} \equiv-\left.\left(\dot{r}_{\mathrm{GW}} / r\right)\right|_{r_{\mathrm{f}}}$ is a scaled inspiral rate evaluated at the final separation.

The parameter $R$ can also be considered an inverse residence time, which relates to the time spent at each orbital radius during a GW-driven decay. It is useful to express this quantity in terms of the orbital frequency, which provides a dimensionless inspiral rate:

$\frac{R}{\Omega}=\frac{\dot{r}}{\Omega r}=\frac{64}{20 \sqrt{2}} \frac{1}{(1+1 / q)(1+q)}\left(\frac{r_{\mathrm{S}}\left(M_{1}\right)}{r}\right)^{5 / 2}$,

where $r_{\mathrm{S}}=2 G M_{1} / c^{2}$ is the Schwarzschild radius of the primary $\mathrm{SMBH}$ and $\Omega$ is the orbital angular frequency. Note that $\Omega / R$ is approximately the number of orbits a binary spends at each separation $r$ (the total number of orbits from $r$ to 0 is $n_{\text {orb }}=1 /(5 \pi) \Omega / R$ ).

In general, for each simulation, we need to specify an initial and a final position $\left[r_{i}, r_{f}\right]$ for the inspiraling satellite $\mathrm{BH}$. In practice, this choice is guided by two considerations. First, we wish to simulate E/IMRIs that are in the LISA frequency band, close to merger. Second, while ideally we would follow the inspiral for the entire duration of a LISA observation (4 years by default; see below), in practice, we are limited by numerical considerations to a fixed number of orbits ( $N_{\text {orb }}=5000$ unless specified otherwise).
The parameterisation above allows us to conveniently implement these choices, by specifying values for $R_{\mathrm{f}}$ and $t_{\text {tot }}$. These translate to ranges of $\left[r_{i}, r_{f}\right]$ which depend on $q$ and the total binary mass. Motivated by modeling potential LISA sources, we impose inspiral rates $R_{\mathrm{f}}$ corresponding to the final stages of a $q=10^{-3}$, $M_{1}=10^{6} M_{\odot}$ fiducial IMRI, reaching $r_{f}=3 r_{\mathrm{S}}$. Each of our simulations spans $n_{\text {orb }}=5,000$ binary orbits. Provided a final separation $r_{\mathrm{f}}$ and a total number of orbits $n_{\text {orb }}$, one can solve for the initial separation of the binary through the relation

$N_{\text {orb }}=\frac{1}{2 \pi} \int_{r_{\mathrm{i}}}^{r_{\mathrm{f}}} \frac{\Omega}{\dot{r}_{\mathrm{GW}}} d r$.

With our fiducial parameters listed above, our initial binary separation is $r_{i}=8 r_{\mathrm{S}}$.

While the simulation is scale-free (i.e. $r_{0}=\Sigma_{0}=1$ in code units), prescribing an inspiral rate with Eq. 3 implies a physical length scale (i.e. a physical value for $r_{0}$, or equivalently a value of $r_{0}$ in Schwarzschild units). We illustrate this conversion in Figure 2, where we show curves of constant $\dot{r} / \Omega r$ as a function of $q$ and separation $r$ (or corresponding GW frequency $f_{\mathrm{GW}}$ shown on the upper $\mathrm{x}$-axis). We delineate the simulated ranges of binary separations (or equivalently frequency ranges) with straight horizontal lines. Light and dark lines correspond to six different simulations we performed, each spanning 5,000 orbits and probing various physical regimes of coalescence. The dashed portion of the lines indicates overlap, where two different simulations probe the same inspiral rates.

As shown in Fig. 2, simulations with different $q$ cover the same range of dimensionless inspiral rates $\left(7 \times 10^{-6} \leq R_{\mathrm{f}} \leq 2 \times 10^{-4}\right)$, despite the fact that when decreasing the mass ratio, such rates correspond to unphysically small separations inside the innermost stable circular orbit (ISCO). While these inspiral rates are not realised in nature for low- $q$ binaries, this academic exercise allows us to isolate the effect of the inspiral on the torque, changing one parameter at a time, since we expect torques to be sensitive to the inspiral speed in addition to mass ratio.

In addition to the fiducial simulation that starts at $8 r_{\mathrm{S}}$ and reaches the ISCO at $3 r_{\mathrm{S}}$, we ran a set of "slow" simulations (lighter lines in Fig. 2) which probe the $q=10^{-3}$ inspiral from $10 r_{\mathrm{S}}$ to $6.5 r_{\mathrm{S}}$, similar to the range covered in Paper I. Rather than simulating a full 10,000 orbits, we split our simulations into two runs that overlap in inspiral rate. This provides a sanity check that our results are physical, and not dependent on transients introduced by the initial conditions (in other words, we can test whether the end of the "slow run" yields the same torques as the beginning of the "fast" run). Moreover, this reduces the span of radii covered in each single simulation, allowing us to avoid the $\mathrm{BH}$ starting too close to the outer boundary. Each simulation exceeds a viscous time for the disc, which for reference we define as a function of orbital time $t_{\text {orb }}$ at the $\mathrm{BH}$ position as

$t_{\mathrm{visc}}=\frac{2}{3} \frac{r^{2}}{v} \approx 1415\left(\frac{\mathcal{M}}{20}\right)^{2}\left(\frac{\alpha}{0.03}\right)^{-1} \mathrm{t}_{\mathrm{orb}}$.

We neglect relativistic effects and keep the potential Newtonian, despite the fact that we are simulating regions close to the ISCO, where relativistic effects will affect the dynamics. This choice was made primarily for simplicity and to maintain the scale-free nature of the simulation.

We compute the torques exerted by the gas on the secondary $\mathrm{BH}$, but we neglect their effect on the BH's orbital evolution. This assumption is justified in the regime where the disc mass is insignificant compared to the mass of the secondary $\mathrm{BH}$, and when the torque due to $\mathrm{GW}$ emission is far dominant. We demonstrate 


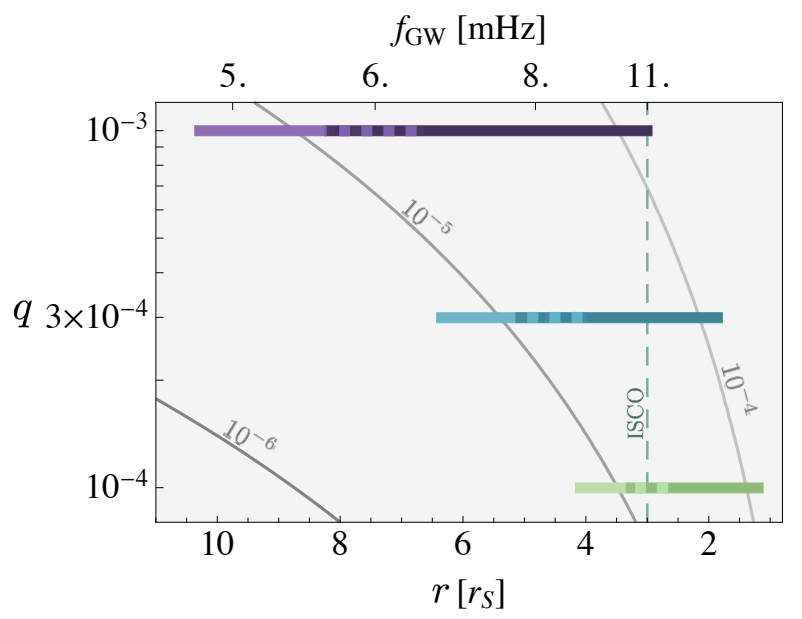

Figure 2. Contour lines (in grey) of constant $\dot{r} /(\Omega r)$, a dimensionless inspiral rate, as a function of $q$ and binary separation $r$ (or corresponding gravitational wave frequency $f_{\mathrm{GW}}$, shown on the upper $\mathrm{x}$-axis for a fiducial primary mass $\left.M_{1}=10^{6} M_{\odot}\right)$. The horizontal colored lines show the ranges of inspiral rates covered in six of our simulations. For each mass ratio, these correspond to different separations in Schwarzschild radii. Because we chose to simulate all three mass ratios over the same dimensionless inspiral rate, portions of the two lower mass ratio simulations correspond to separations inside the ISCO (vertical dashed line).

in $\S 4$ below that both of these criteria hold to high accuracy. This approach keeps the equations scale invariant and $\Sigma_{0}$ arbitrary, significantly reducing our computational costs and allowing us to run a full parameter study (see $\$ 3.5$ ).

\subsection{Sink prescription}

Accretion onto the secondary is implemented with the same approach as in Derdzinski et al. (2019). The gas inside the smoothing radius $\epsilon$ is approximated as a mini-disc with the same $\alpha$ and $\mathcal{M}$ as the global disc. The surface density within a distance $\epsilon$ of $M_{2}$ is decreased on the viscous timescale, $t_{\mathrm{visc}}(\epsilon)$ at a rate

$\frac{d \Sigma}{d t}=-\frac{\Sigma}{t_{\mathrm{visc}}(\epsilon)} \exp \left\{-\left(r_{2} / \epsilon\right)^{4}\right\}$.

As discussed in Paper I, the chosen parameters for a thin-disc accretion model correspond to an accretion rate that can be well in excess of the Eddington rate, depending on the normalisation of the disk surface density. Gas within the sink radius is depleted at a rate $\dot{M} \approx \pi \epsilon^{2} \Sigma_{\text {sink }} / t_{\text {visc }}$, where $\Sigma_{\text {sink }} \sim 10^{3} \Sigma_{0}$ due to the stark increase in density of gas near the $\mathrm{BH}$ for $q=10^{-3}$ (Fig. 1). Converting this to a physical rate with $M_{2}=10^{3} M_{\odot}, r_{0}=3 r_{\mathrm{S}}, \mathcal{M}=20$, and $\alpha=0.03$ then implies an accretion rate $\dot{M} \gtrsim \dot{M}_{\text {Edd }}$ for $\Sigma_{0} \gtrsim 10 \mathrm{~g} \mathrm{~cm}^{-2}$, given $\dot{M}_{\mathrm{Edd}}=4 \pi G M_{2} /\left(\epsilon_{\mathrm{eff}} \kappa_{\mathrm{es}} c\right)$, where $\kappa_{\mathrm{es}}=0.4 \mathrm{~cm}^{2} \mathrm{~g}^{-1}$ is the electron scattering opacity and we assume a radiative efficiency $\epsilon_{\mathrm{eff}}=0.1$. This is a common shortcoming for many accretion disc simulations; it is computationally challenging to simulate highly supersonic discs, and thus we are often limited to unrealistically low Mach numbers.

For an additional reference, if we compare the prescribed accretion rate to a Bondi rate $\dot{M}_{\text {Bondi }}=4 \pi G^{2} M_{2}^{2} \rho_{0} / c_{s}^{3}$ (e.g. Edgar 2004) assuming the relative velocity between the $\mathrm{BH}$ and gas is zero (as it is comoving), we find that the accretion rate is well below the Bondi rate $\left(\dot{M}_{\text {sink }} / \dot{M}_{\text {Bondi }} \sim 10^{-6}\right)$ for any choice of $\Sigma_{0}$. This suggests that depending on how the accretion flow actually occurs, even higher rates may be feasible.

Nevertheless, we consider our chosen accretion prescription to be relatively "slow" when compared to others in the literature that deplete gas within the sink on $\sim$ orbital timescales. In our case, gas is only partially depleted within the sink radius and only for the highest simulated mass ratio $q=10^{-3}$. Unlike for more nearly equal-mass binaries (such as those studied in Duffell et al. 2020), we find here that the sink prescription affects the torques for a sufficiently massive gap-opening satellite. We discuss this further in Section 4.

\subsection{Torque measurement}

Here we describe how the gas disc torque is computed in the simulations and define other torques for comparison.

When comparing torques on binaries of different mass ratios, it is useful to utilize the Type I formula from Tanaka et al. (2002),

$T_{0}=\Sigma(r) r^{4} \Omega^{2} q^{2} \mathcal{M}^{2}$,

where $\Sigma(r)$ is the initial surface density profile. As the BH migrates and the disc evolves from its initial conditions, it is useful to normalise the measured torque by $T_{0}$ in order to scale out the expected radial dependence of the torque. Analysing a dimensionless torque allows us to compare results for different mass ratios and isolate the effect of the inspiral.

The dominant mechanism for angular momentum loss on the secondary is the torque due to GW emission,

$T_{\mathrm{GW}}=\frac{1}{2} M_{2} r \dot{r}_{\mathrm{GW}} \Omega$.

Note that this is the torque on only one component of the binary.

The primary focus of this work is the gravitational torque (also referred to as migration or gas torque) $T_{\mathrm{g}}$, which arises from the gravitational force exerted by the gas on the secondary BH. We calculate this torque by summing up the $\phi$-component of the gravitational force $\mathbf{g}_{\phi}$ crossed with the binary lever arm $\mathbf{r}$ over all the grid cells in the disc,

$T_{\mathrm{g}}=\sum\left|\mathbf{g}_{\phi} \times \mathbf{r}\right|$

where $|\mathbf{r}|$ is the distance from the secondary to the center of mass of the binary, which in our setup (and in the limit of $q \ll 1$ ) is at the origin. We also discuss the torque density, defined by $\mathcal{T} \equiv \mathbf{g}_{\phi} \times \mathbf{r}$ before summation, to analyse contributions from different patches of the disc to the total torque.

Torque can also be imparted via accretion of gas that directly adds both mass and momentum to the secondary BH (sometimes called accretion torque). For our parameters we find this torque to be significantly weaker than the gravitational torque (by several orders of magnitude, as in Derdzinski et al. (2019), so we refrain from discussing it further in the present work.

The magnitude of gas torques (Eqs. 9 and 11) all depend linearly on the normalisation of the surface density, which is arbitrary in our simulation setup. Rather than choosing a single value for $\Sigma_{0}$, we will use this freedom of normalisation to ask: at what value of the surface density do gas torques produce a detectable phase drift in LISA's GW measurements? Additionally, is this density physically reasonable, given the densities expected in accretion disc models?

Estimates for accretion disc densities in the vicinity of the central SMBH vary by several orders of magnitude. We adopt two limiting estimates for the surface density normalisation $\Sigma_{0}$ (at the secondary's final radius, taken to be $r_{\mathrm{f}}=3 r_{\mathrm{S}}$ for our detectability 
estimates below) for the inner regions of thin, near-Eddington accretion discs. They depend on the presumed accretion rate $\dot{M}$ and the viscosity parameter $\alpha$. The models differ in whether the viscosity scales with the gas pressure or with the total (gas + radiation) pressure, a choice that has a large impact when relating the accretion rate to $\Sigma_{0}$. Lower viscosity discs require much higher surface densities to maintain the same accretion rate.

We normalise our disc densities to represent AGN accreting at $10 \%$ of the Eddington rate with a radiative efficiency of $10 \%$. The first estimate is obtained from the seminal model for a thin, viscous accretion disc (i.e. $\alpha$-disc; Shakura \& Sunyaev 1973). In the inner, radiation-pressure dominated region,

$\Sigma_{\alpha}=41.08\left(\frac{\alpha}{0.03}\right)^{-1}\left(\frac{\dot{M}}{0.1 \dot{M}_{\text {Edd }}}\right)^{-1}\left(\frac{r}{3 r_{\mathrm{S}}}\right)^{3 / 2} \mathrm{~g} \mathrm{~cm}^{-2}$.

In case the viscosity is proportional only to the gas pressure (i.e. for a so-called $\beta$-disc), the surface density at the same accretion rate is several orders of magnitude higher,

$$
\begin{aligned}
\Sigma_{\beta}=2.11 \times 10^{7}\left(\frac{\alpha}{0.03}\right)^{-4 / 5}\left(\frac{\dot{M}}{0.1 \dot{M}_{\mathrm{Edd}}}\right)^{3 / 5} \times \\
\times\left(\frac{M}{10^{6} M_{\odot}}\right)^{1 / 5}\left(\frac{r}{3 r_{\mathrm{S}}}\right)^{-3 / 5} \mathrm{~g} \mathrm{~cm}^{-2},
\end{aligned}
$$

see Haiman et al. (2009). While both of these models carry a different radial density scaling than our disc model, the values are meant to provide a reference for possible surface densities, which becomes important for our detectability estimates in $\$ 5$ below. Note that in both these estimates the total disc mass inside the BH's orbit is much less than the mass of the BHs. For example, even with the high-end estimate, an integral of the total enclosed mass within $10 r_{\mathrm{S}}$ yields a mass as low as

$M_{\mathrm{encl}}=2 \pi \int_{3 r_{\mathrm{S}}}^{10 r_{\mathrm{S}}} \Sigma_{\beta} r d r \simeq 0.16 M_{\odot}$

for $\alpha=0.03, M=10^{6} M_{\odot}$, and $\dot{M}=0.1 \dot{M}_{\mathrm{Edd}}$.

\subsection{Simulation Suite}

In Paper I, we performed a simulation for a single system with $q, \alpha, \mathcal{M}=10^{-3}, 0.03,20$, over a range of dimensionless inspiral rates between $7 \times 10^{-6}$ and $2 \times 10^{-5}$. We extend on that study by expanding the range of inspiral rates to higher values up to $\dot{r} / \Omega r=2 \times 10^{-4}$, and then varying $q, \mathcal{M}$, and $\alpha$. Our fiducial system is a $q=10^{-3}$ binary embedded in a disc with $\alpha=0.03$ and $\mathcal{M}=20$. We then run simulations for three different mass ratios $q=10^{-3}, 3 \times 10^{-4}, 10^{-4}$, each with three different values of $\alpha=0.01,0.03,0.1$. We also run simulations with a range of Mach numbers $\mathcal{M}=10,20,30$ around our fiducial model. In total, we present 15 different simulations, which are listed in Table 1, where each run is labeled with its mass ratio and viscosity (for example, 'q1e3a03' corresponds to a run with $q=10^{-3}$ and $\alpha=0.03$.)

For computational feasibility, our study is limited to higher values of $\alpha$ and lower Mach numbers, in order to avoid prohibitively long viscous times to reach steady state (see Eq. 7). Our fiducial choice of $\mathcal{M}=20$ corresponds to a much hotter and thicker disc than expected in thin, near-Eddington AGN discs, where continuum emission suggests Mach numbers exceeding $\mathcal{M} \sim 100$ Krolik 1999). However, higher Mach numbers are numerically challenging to simulate, primarily because highly supersonic flows require increasingly high resolution as they develop complicated, small scale features. As we show in $\$ 4.1 .3$ below, increasing the Mach number to 30 already produces very noisy torques and a dense and unstable gas morphology.

\section{RESULTS}

Here we describe results from our simulations - measuring and analysing the torques exerted on the satellite $\mathrm{BHs}$ - before deriving estimates of the detectability of the corresponding imprints in the LISA GW waveforms.

\subsection{Gas torques depend on parameters}

As predicted by analytical estimates such as $T_{0}(r, q, \mathcal{M})$, we expect that torques will not only depend on system parameters but also evolve with radius as the binary separation decreases. We find that the magnitude of gas torques generally agrees, within an order of magnitude, with the analytical predictions $T_{0}(q, \mathcal{M}, r)$ (Eq. 9). However, the direction of the torque is difficult to predict. Torques tend to oscillate around a value close to (typically less than) $T_{0}$, but at the late stages of the inspiral, the evolution may deviate from the expected scaling. As we discuss in the following sections, whether torques are negative (inward) or positive (outward) depends on the combination of $q, \alpha$, and $\mathcal{M}$, and we find there is no direct or obvious scaling in this intermediate mass ratio regime.

\subsubsection{Mass ratio}

In Fig. 3, we plot the measured $T_{g} / T_{0}$ as a function of inspiral rate (Eq. 5, itself a function of $r$ ) measured in simulations with different mass ratios in a disc with fiducial parameters $\alpha=0.03$ and $\mathcal{M}=20$. As we expect from similar studies of torques on stationary (i.e. non-migrating) satellites, torques in this regime are sensitive to even small changes in system parameters. In fact, when increasing $q$ from $10^{-4}$ to $10^{-3}$, the figure shows that the direction of the torque changes. This particular behavior is, however, also dependent on the choice of $\alpha$ (see below).

Additionally, the torque exerted on the $q=10^{-3}$ satellite is significantly more noisy compared to the smaller mass ratios. This is attributed to the large pile-up of gas that accumulates very close to the $\mathrm{BH}$ in this mass regime. We discuss this behavior and its consequences in $\S 4.2$ below.

First we compare the strength of the gas torque to GWs. We compute the average torque from the last 2000 orbits in each of the slower-inspiral runs (where the scaled torques $T_{g} / T_{0}$ are constant to a good approximation), and compare these to the $\mathrm{GW}$ torques during the last stages of coalescence. These comparisons are shown in Fig. 4. The average torque measured in the simulation is extrapolated to earlier times for the two smaller mass ratios, assuming that the scaled torque remains constant (shown by the dashed curves). To scale the torques to physical values, we normalise the surface density with the $\beta$-disc estimate (Eq. 13), making these high-end estimates of the disc torques. In all cases, despite the high assumed disc density, gas torques are several orders of magnitude weaker than that due to GWs at this stage of coalescence. However, as we will show in $\$ 5$, over an observation of many thousand cycles even weak gas impact can accumulate and produce detectable signatures in the measured GW signal.

In most cases we explore here, the average $T_{g}$ is within a factor of a few of the analytical estimate $T_{0}$, but deviates towards stronger torques (either negative or positive) as the inspiral rate increases. 
Simulations

\begin{tabular}{|c|c|c|c|c|c|}
\hline Name & Mass Ratio & Viscosity & Mach Number & Separation $\left[r_{\mathrm{S}}\right]$ & Average Torque \\
\hline & $q$ & $\alpha$ & $\mathcal{M}$ & {$\left[r_{i}, r_{f}\right]$} & $\left\langle T_{\mathrm{g}} / T_{0}\right\rangle$ \\
\hline $\begin{array}{l}\text { q1e3a03 } \\
\text { (fiducial) }\end{array}$ & $10^{-3}$ & 0.03 & 20 & $\begin{array}{c}10.3,6.5] \\
{[8.2,3.0]}\end{array}$ & $\begin{array}{l}0.19 \\
\text { *see Fig. } 3\end{array}$ \\
\hline q3e4a03 & $3 \times 10^{-4}$ & 0.03 & 20 & $\begin{array}{l}{[6.4,4.0]} \\
{[5.1,1.9]}\end{array}$ & $\begin{array}{l}0.01 \\
\text { *see Fig. } 3\end{array}$ \\
\hline q1e4a03 & $10^{-4}$ & 0.03 & 20 & $\begin{array}{l}{[4.1,2.6]} \\
{[3.3,1.2]}\end{array}$ & $\begin{array}{l}-0.29 \\
* \text { see Fig. } 3\end{array}$ \\
\hline q1e3a1 & $10^{-3}$ & 0.1 & 20 & {$[8.2,3.0]$} & 0.34 \\
\hline q3e4a1 & $3 \times 10^{-4}$ & 0.1 & 20 & {$[5.1,1.9]$} & 0.38 \\
\hline q1e4a1 & $10^{-4}$ & 0.1 & 20 & {$[3.3,1.2]$} & -1.26 \\
\hline q1e3a01 & $10^{-3}$ & 0.01 & 20 & {$[8.2,3.0]$} & $\sim 0.01$ (*see Fig. 5) \\
\hline q3e4a01 & $3 \times 10^{-4}$ & 0.01 & 20 & {$[5.1,1.9]$} & $\sim-0.51(*$ see Fig. 5) \\
\hline q1e4a01 & $10^{-4}$ & 0.01 & 20 & {$[3.3,1.2]$} & $\sim-0.25$ (*see Fig. 5) \\
\hline q1e3a03m10 & $10^{-3}$ & 0.03 & 10 & {$[8.2,3.0]$} & -0.30 \\
\hline q1e3a03m30 & $10^{-3}$ & 0.03 & 30 & {$[8.2,3.0]$} & $\sim 0.03$ (*see Fig. 7) \\
\hline q1e3a03 (no sink) & $10^{-3}$ & 0.03 & 20 & {$[8.2,3.0]$} & *see Fig. 9 \\
\hline
\end{tabular}

Table 1. List of our 15 simulations and their parameters. Each binary is forced to inspiral at the GW-driven rate from its initial $r_{i}$ to its final separation $r_{f}$ (listed in Schwarzschild units). In those cases where we ran two simulations for a single binary to probe different inspiral rates, two ranges of radii are listed (first three rows). We also show the average torque value (measured over the last 2000 binary orbits) in simulations for which we calculate the SNR of the gas-induced deviations in the GW waveform. *Depending on the disc and system parameters, some simulations show deviations from the average torque throughout the GW inspiral - these we denote in the last column with a reference to the corresponding Figure.

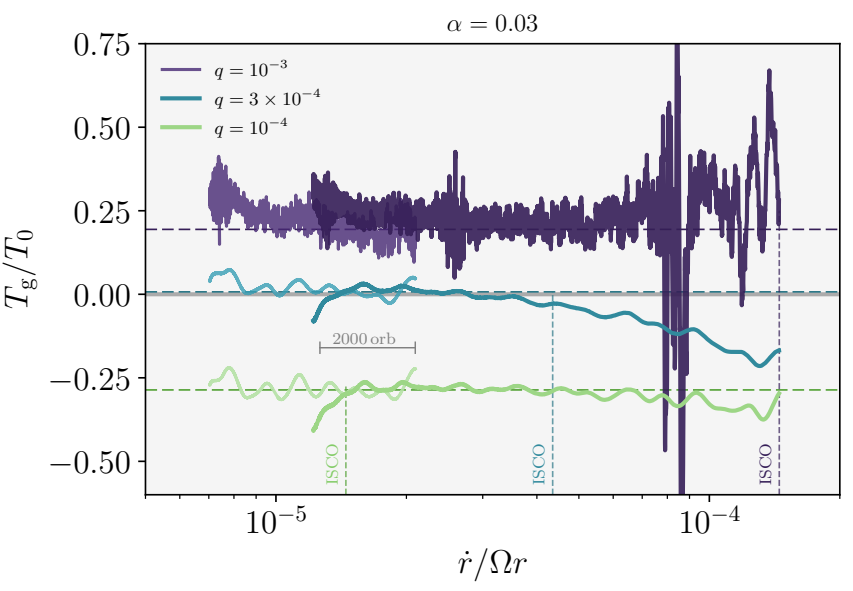

Figure 3. Gas torque (normalized by the Type I torque $T_{0}$ ) as a function of dimensionless inspiral rate. Each color corresponds to a different mass ratio, and each mass ratio is covered by two simulations overlapping in the inspiral rates (lighter lines on the left, corresponding to slower inspiral, and darker on the right, corresponding to faster inspiral). Dashed horizontal lines show the average normalised torque, measured over the last 2000 orbits of the slower inspiral runs, as marked by the grey bracket. Interestingly, the absolute values of the gas torque are similar in the $q=10^{-4}$ (lowest curves) and $q=10^{-3}$ (uppermost curves), while their signs differ; this appears to be a coincidence. For simulations reaching fast inspiral rates, the torque begins to deviate from the average for the two higher mass ratios. Vertical dashed lines mark which inspiral rate corresponds to the ISCO for each $q$.

This happens at the final stages of the inspiral (the final $\sim 1000$ orbits), and in the case of the smallest mass ratio $q=10^{-4}$, in reality the merger occurs before the inspiral has a chance to substantially affect the torque. However, as we describe below, when or whether this deviation occurs depends on other disc properties.

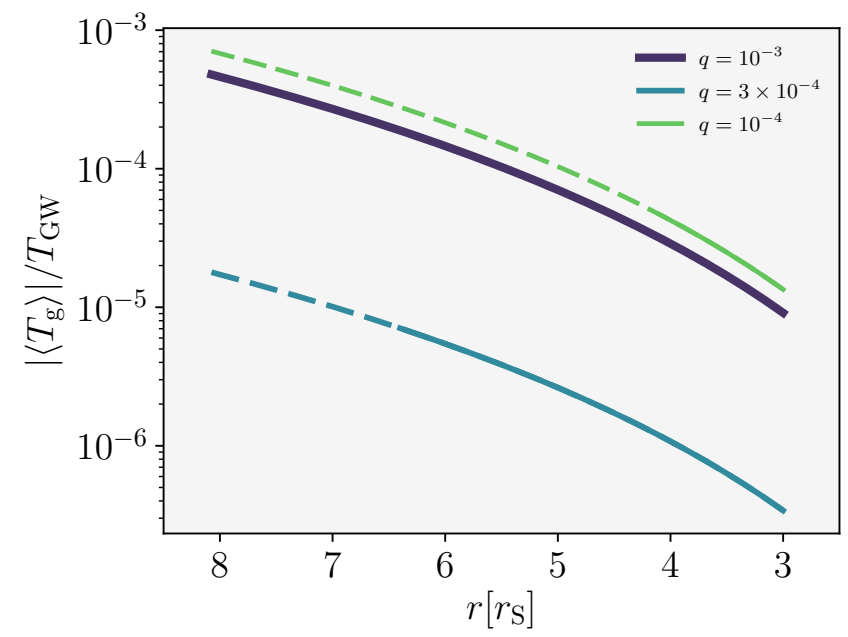

Figure 4. Average gas torques from simulations with three different mass ratios with $\alpha=0.03$ and $\mathcal{M}=20$ (shown in Fig. 3), scaled by the GW torques during the last few $r_{\mathrm{S}}$ prior to ISCO. The dashed portion of each curve indicates the radii for which the torque is extrapolated outside of the simulated range (see Fig. 2). Gas torques are normalized by $\Sigma_{\beta}$, a high-end estimate for the disc surface density (Eq. 13). Gas torques are significantly weaker than GWs in this regime, but can accumulate to a detectable effect over the large number of cycles in the LISA band (see §5).

\subsubsection{Viscosity}

In this highly nonlinear regime, viscosity affects several important aspects to this system, each of which contribute to the torque in significant ways. First, viscosity affects the gap depth, which will affect the magnitude of the Lindblad torque excited in the disc. Secondly, viscosity sets the rate that angular momentum can be carried away from the corotation resonances, and whether corotation 
torques are in a "saturated" or "desaturated" state (Masset et al. 2006; Duffell 2015). Third, viscosity can suppress instabilities in the disc that might otherwise generate vortices which exert nontrivial time-dependent torques on the perturber. Finally, in this complicated nonlinear regime, it is fully possible there are comparable additional torque affects that have not been considered yet, as most analysis of viscous affects on torque are carried out in the linear or weakly nonlinear regimes. All of this adds up to the result that the torque has a nontrivial $\alpha$ dependence, and even small changes in $\alpha$ (at the least of order $10^{-2}$, given the values explored in the present work)

can change the sign of the torque. This is seen in simulations of a range of mass ratios from planetary scales (Duffell 2015) up to $q \sim 0.5$ in the context of MBH binaries (Duffell et al. 2020).

We have run additional sets of simulations for each mass ratio in discs with higher and lower viscosities ( $\alpha=0.1$ and $\alpha=0.01$ ) to observe changes in the overall torque and its evolution with the inspiral rate. The results are shown in Fig. 5. We find that the magnitude $\left|T_{\text {gas }}\right|$ typically increases in strength with $\alpha$, as has been observed in other numerical studies (e.g. Robert et al. 2018), although the direction changes unpredictably, particularly for $q=$ $3 \times 10^{-4}$.

Intuitively, we expect that for higher values of viscosity, the inward migration driven by GW emission should have less impact on the torque, simply because the disc can reorganize more quickly (recall that $t_{\mathrm{visc}} \propto \alpha^{-1}$ ), and thus a stronger viscosity leads to a faster radial inflow ${ }^{1}$ ). Indeed, this is what we observe in the top panel of Fig. 5. For $\alpha=0.1$ the torque is essentially constant throughout the entirety of the inspiral (modulo short time-scale oscillations) for all mass ratios, and its absolute value can be approximated by $T_{0}$ to within a factor of two. For the weaker viscosity, the lower panel of Fig. 5 shows that $T_{g} / T_{0}$ deviates from the nearly constant value during earlier stages, leading to an increasingly negative torque.

In the case of thin, fully ionised discs in near-Eddington AGN, observational evidence suggests that viscosity may reach values of $\alpha=0.1-0.4$ (King et al. 2007). Our results therefore imply that E/IMRIs in such viscous discs may be subject to stronger torques, but are less likely to show significant changes in torque during the inspiral. However, it is the combination of $\alpha$ and $\mathcal{M}$ that determines the disc dynamics (note the kinematic viscosity $v \propto \alpha / \mathcal{M}^{2}$ ), and the values we consider here provide AGN-like characteristics (e.g. gap depth). Furthermore, in reality $\alpha$ may scale with radius - thus it is possible that the torque evolution seen in lower $\alpha$ simulations may still be relevant for AGN. In Section 5, we discuss the case in which torques follow a simple scaling with radius.

\subsubsection{Mach number}

The Mach number, a measure of disc temperature and thickness, is also a critical factor in determining the gap depth and disc dynamics near the BH (Duffell 2015). A low Mach number disc is subject to stronger pressure forces, resulting in shallower gaps, while a higher Mach number describes a dynamically colder disc that can consequently form deeper gaps. For high enough Mach number the scale height of the disc (recall $h / r=1 / \mathcal{M}$ ) can approach or fall within the Hill sphere of the perturber, leading to a more dynamic gas flow across the gap (see below).

For our fiducial system with $q=10^{-3}$ and $\alpha=0.03$, we

1 This is further supported by the idea that a higher viscosity will inhibit gap formation, given that more efficient angular momentum transport leads to a rapid replenishing of gas in the co-orbital region (Armitage 2007). explore a range of Mach numbers from $\mathcal{M}=10-30$. While this range is limited, we are able to observe trends in gap depth and gain insight into the dependence of the gas dynamics close to the $\mathrm{BH}$ on $\mathcal{M}$, which gets increasingly complex for colder discs.

Fig. 6 shows surface density contours of gas close to the $\mathrm{BH}$ at the end of each simulation. For the lowest Mach number $(\mathcal{M}=10$, which, we note, is a value often adopted in binary simulations), pressure forces significantly smooth the flow. For the highest Mach number $(\mathcal{M}=30)$, gas flows more tightly around the $\mathrm{BH}$ (indeed, the scale height and corresponding smoothing length $\epsilon$ is smaller). Gas morphology within the Hill radius is dynamic, with narrower streams that flow across a deeper gap and stark density contrasts that lead to instabilities in the gap edges and streams.

Fig. 7 shows the corresponding gas torques. In the warmer disc ( $\mathcal{M}=10$; purple curve), the torque is smoother and negative, rather than positive as in the fiducial $\mathcal{M}=20$ case (blue curve). For the colder disc ( $\mathcal{M}=30$; blue curve), the net torque becomes positive, but the most striking feature is that the dynamic flow around the $\mathrm{BH}$ produces a highly variable torque. The variability increases dramatically as the $\mathrm{BH}$ migrates inward.

In order to verify that the large fluctuations are physical and not due to under-resolved gas flow near the $\mathrm{BH}$, we performed higherresolution runs as a test (up to 800 radial cells, corresponding to $\Delta r \gtrsim h / 10)$. These tests show that the variability persists, but the amplitude and timescale are not yet converged. In fact, a higher resolution leads to larger amplitude in torque fluctuations. This leads us to believe that the fluctuations are physical, and that highly supersonic discs are indeed more sensitive to the evolution of an embedded $\mathrm{BH}$ during a $\mathrm{GW}$ inspiral. Without stronger pressure forces to smooth out fluctuations in the flow, gas torques become more unstable. A more detailed investigation of the impact of such a variable torque on a GW inspiral will be investigated in future work.

\subsection{Dissecting the torque}

With our simulations we have the ability to assess contributions of different parts of the disc to the net gravitational torque. Of particular interest is being able to distinguish between gas very close to the satellite $\mathrm{BH}$, i.e. near and within its Hill radius, and that from elsewhere in the disc (the inner and outer discs as well as streams crossing the gap). One might compare this to corotation torques discussed in the planetary migration literature, keeping in mind that these studies concern gas within the entire co-orbital region rather than just the Hill sphere. Similar to what we find here, numerical studies find that corotation torques can become positive in discs with steep density gradients (Paardekooper \& Papaloizou 2008) or with sufficiently high viscosities (Masset et al. 2006; Duffell 2015). An important distinction to note is that corotation torques include streams of gas in the horseshoe region, which includes the entire annulus of the migrator and often excludes the Hill radius.

Here, we do not consider gas within the entire co-orbital annulus of the $\mathrm{BH}$, because most of this gas is at low densities due to gap-opening. Instead we focus on the gas within the Hill radius of the secondary. In our case, streams in this region that flow across the gap are both dense and close to the $\mathrm{BH}$, and thus dominate the "corotation" torque. As we show, this contribution to the torque arises from a back-to-front asymmetry in the gas morphology at or inside the Hill radius.

The Hill radius, defined as the region where the orbital velocity of gas bound to the satellite $\mathrm{BH}$ matches the orbital velocity of the 

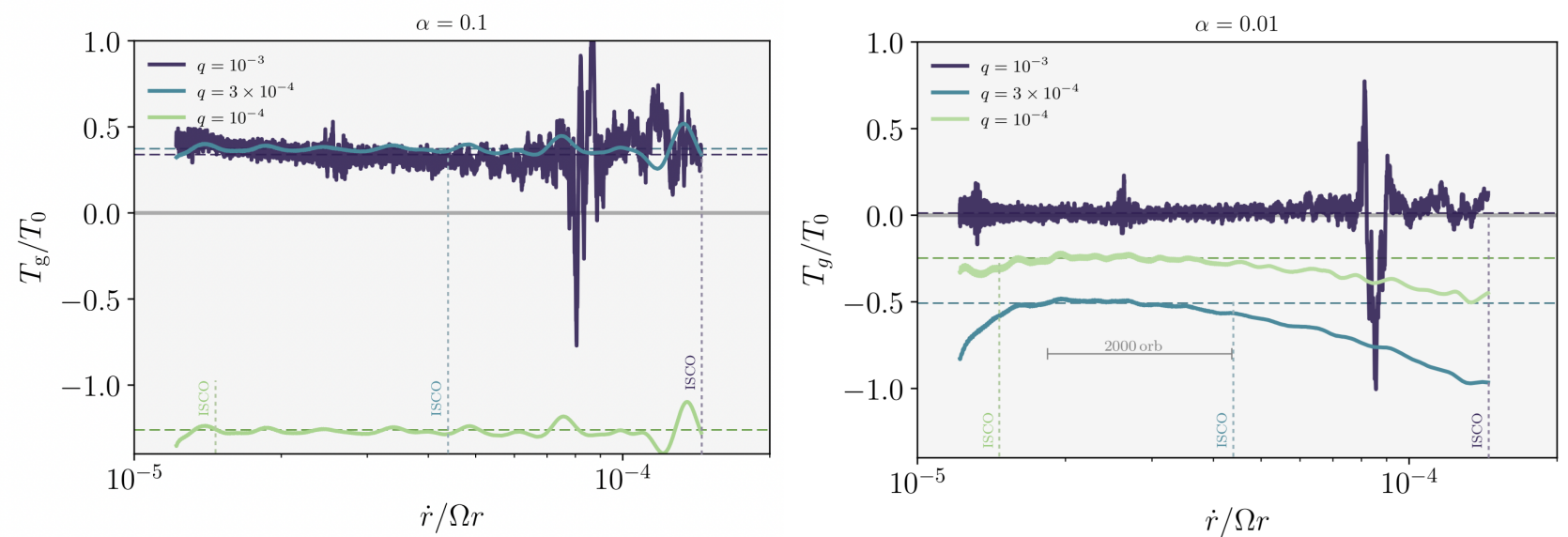

Figure 5. Left panel: Gas torque normalized by $T_{0}$ for three different mass ratios, in a disc with $\alpha=0.1$. Higher viscosity means the disc stabilises more quickly, and torques do not change as significantly as the inspiral accelerates. Right panel: Gas torque normalized by $T_{0}$ for runs with $\alpha=0.01$. Dashed horizontal lines in both panels indicate an average taken over a 2000-orbit window depicted by the grey bracket.
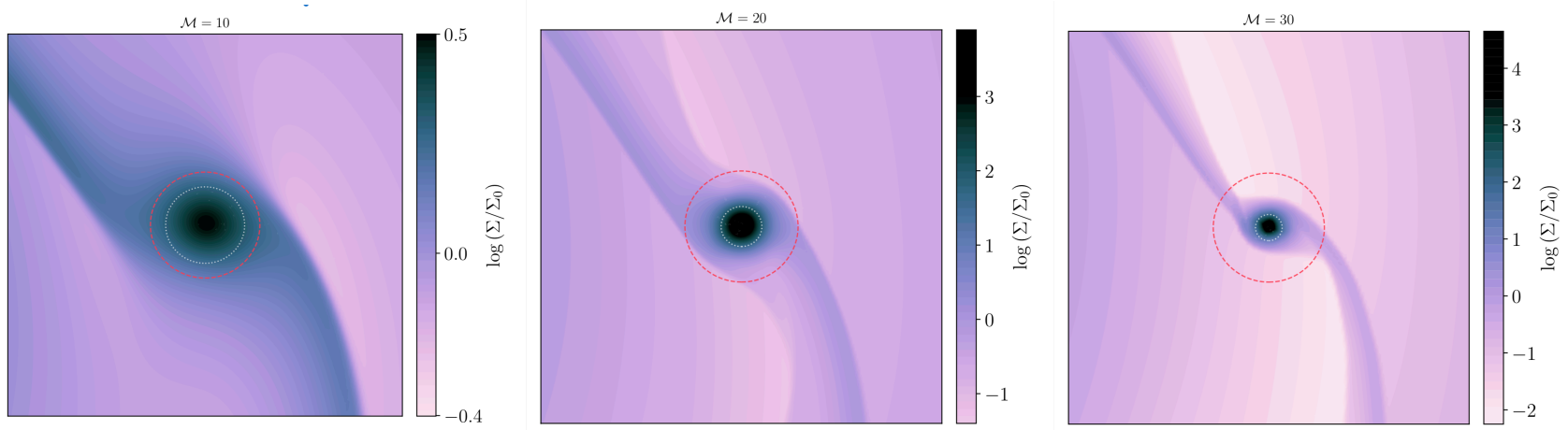

Figure 6. Snapshots of the surface density, zooming in on the region close to the BH. for three different Mach numbers, as labeled on the top of each panel. For comparison we show the Hill sphere (red circles) and the disc scale height (dashed yellow circles). Lower Mach number discs are hotter and subject to stronger pressure forces that smooth out the flow. For higher Mach numbers, as the scale height becomes smaller than the Hill sphere, gas flow across the gap occurs along thinner streams and the $\mathrm{BH}$ carves a much deeper gap. Note the different color scalings in each of the panels; the surface densities in the $\mathcal{M}=30$ case are several orders of magnitude higher.

BH itself, is given by

$r_{\mathrm{H}}=\left(\frac{q}{3}\right)^{1 / 3} r$.

As discussed in Paper I already for the $q=10^{-3}$ case, gas pile-up close to the $\mathrm{BH}$ becomes non-negligible. This can be seen in the density contrasts in Fig. 6, or in Fig. 8 where we show various distributions of torque density $\mathcal{T}$ in our fiducial runs for each $q$. Excising the Hill radius (top panels) allows us to observe contributions to the torque from nearby streams, while zooming in on the Hill radius (bottom panels) allows us to analyse the dominant torque contributions (due to its proximity to the $\mathrm{BH}$ ). Most notable is the high torque density for $q=10^{-3}$ arising from the highdensity atmosphere that accumulates around the BH. Despite such high values of $\mathcal{T}$, the net torque in this region remains below $T_{0}$, implying that as density of the circum-BH gas increases, so does its degree of front-to-back symmetry. This is likely a symptom of gas accumulating within a deeper potential well around a more massive secondary $\mathrm{BH}$, which naturally results in a more compact (yet still asymmetric) distribution.

Gas within the Hill radius contributes a substantial fraction to the total torque, as we show in Fig. 9. This figure shows separately the torque contributions from gas within and outside of the Hill radius for the fiducial $q=10^{-3}$ run. Gas inside $r_{\mathrm{H}}$ is responsible for the positive component of the torque and also shows the strongest dependence on the inspiral rate, increasing from $\sim 4 r_{\mathrm{S}}$ to $3 r_{\mathrm{S}}$ (see the solid lines in Fig. 9). This region is sometimes assumed to not contribute to the torque, or is damped by a manual "tapering" function (see, e.g. Dempsey et al. 2020), even though this gas is a crucial component of material flow across the gap and may exhibit non-negligible asymmetries. Indeed, in simulations by Crida et al. (2009), the migration rate of a live planet was found to be dependent on how much the torques from the material inside the Hill sphere are damped, suggesting that this gas is a significant contributor to the net torque, and indeed a physical component that should not be excluded.

In the present work, the Hill sphere torque is of particular importance as the asymmetry near the BH may be exacerbated during a sufficiently fast inspiral (here driven by GWs). We expect that any changes in the torque during a GW-driven inspiral will first arise from gas closest to the BH. This is shown more clearly by comparing versions of our fiducial run for which the sink prescription 


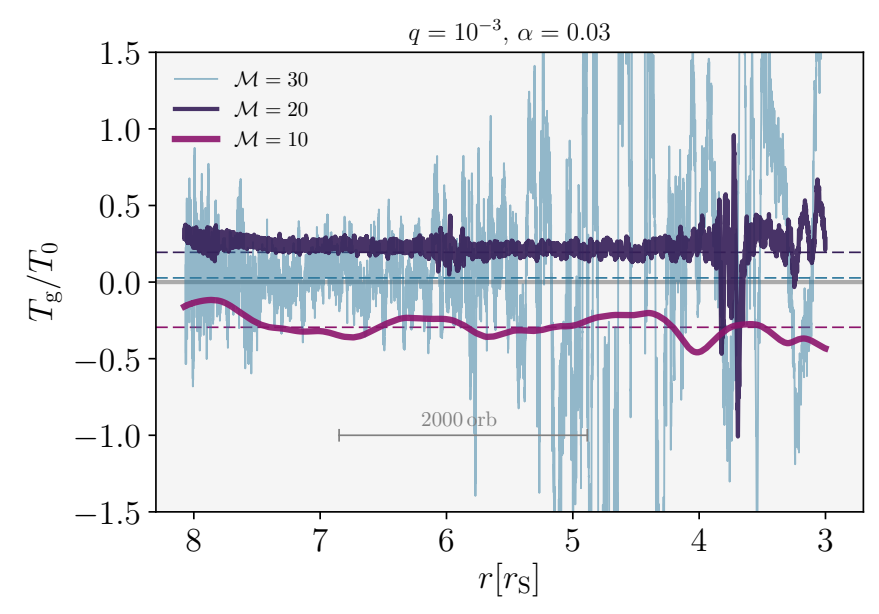

Figure 7. Gas torque on a $q=10^{-3}$ inspiral in a disc with $\alpha=0.03$, for three different Mach numbers. Dashed lines show averages taken over the depicted 2000 orbit window. With higher Mach number, the torque shows large variability throughout the inspiral, with amplitude increasing especially at the later stages.

is turned off (shown as light curves in Fig. 9). Unsurprisingly, an accreting $\mathrm{BH}$ experiences less positive torque as density within the Hill radius is depleted (while it is nearly unaffected outside). Without accretion, torques from within $r_{\mathrm{H}}$ substantially increases with the inspiral rate, more than doubling in comparison to $T_{0}$ within the final 5,000 orbits of the inspiral (from $8 r_{\mathrm{S}}$ to $3 r_{\mathrm{S}}$ ). Conversely, we expect that for arbitrarily fast accretion rates (e.g. if the $\mathrm{BH}$ manages to continually deplete gas in the Hill region), the positive contribution to the torque would diminish and the $\mathrm{BH}$ would instead feel a stronger, negative torque exerted by the rest of the gas in the disk.

For satellites with mass ratios below $q=10^{-3}$ accretion has a negligible impact on the torque - when comparing runs with and without accretion, we see no distinct difference in the surface density profile or the torque, given that smaller satellites accrete an insignificant amount of material according to our sink prescription.

The sensitivity to accretion prescription observed for $q=10^{-3}$ raises the possibility that for IMRIs, detecting torque evolution with frequency may provide insight into the gas dynamics near the $\mathrm{BH}$. We hypothesize that this accretion dependence would occur for higher $q \gtrsim 10^{-3}$, although this regime remains to be explored. However, we note that for mass ratios above $q \sim 0.04$, the gas dynamics transitions from an annular gap to a circumbinary cavity (D'Orazio et al. 2016). In this regime, recent simulations confirm that the sink rate does not affect the torque (Duffell et al. 2020). Interestingly, the latter work finds that between $0.01<q<0.03$ the torque follows the trend we see here: fast accretion rates result in a stronger, more negative torque.

\subsection{Torque evolution}

Does the torque on an embedded IMRI change in response to the increasingly rapid inspiral? This is important as a physics question about the system, as well as a practical question about interpreting any future LISA data. Additionally, it is important to know whether the numerous previous studies of disc torques for non-migrating satellites hold for GW-driven E/IMRIs, or if the torques are modified and need to be re-computed in simulations that include the GWdriven inspiral.
The short answer is: it depends. Whether the GW-driven inspiral produces a significant effect on the torque depends on the mass of the inspiraling $\mathrm{BH}$, the disc viscosity, Mach number, and the $\mathrm{BH}$ accretion efficiency.

During the inspiral, we observe three effects that are possibly correlated with the nonzero inspiral rates. First, for all simulated binaries, the torque on a migrating $\mathrm{BH}$ shows long-term oscillations (on a timescale of $\sim 100$ s of orbits). These are most clearly seen in Fig. 3 for $q=3 \times 10^{-4}$ and $q=10^{-4}$. Despite differing mass ratios these oscillations occur on similar timescales, which suggests they are caused by the same mechanism, likely global perturbations induced by the moving perturber. Although long-term modulations have been seen in the accretion rates of non-inspiraling, near-equal-mass binaries (e.g. see Fig.11 in D'Orazio et al. 2013), these modulations have not been seen for $q \ll 1$. In our case, the long-term modulations in the torque arise from global perturbations as they are present in the torque contribution from outside the Hill radius. The magnitudes are of order $\lesssim 10 \%$, so for detectability purposes the torque can be approximated by an average, or possibly a stationary approximation for Type II torques. However, analytical approximations neglect the ability for Type II torques to become positive.

Second, depending on the mass ratio, a sufficiently fast inspiral rate can change the magnitude of the torque. These changes arise from changes in the gas distribution inside the Hill radius. For our fiducial viscosity $\alpha=0.03$ (Fig. 3), the torque begins to deviate from its steady-state value ${ }^{2}$ at inspiral rates approaching $\dot{r} / \Omega r \sim 10^{-4}$. In reality, this rate is only reached in a circular binary when $q \geq$ $10^{-3}$. Lower mass ratio binaries merge before reaching this rate. However, this trend is dependent on accretion efficiency and disc viscosity. In discs with lower viscosity (or less efficient accretion onto the secondary), we expect this deviation to occur at earlier times. Overall we find that the most prominent torque evolution occurs for a relatively massive satellite with $q=10^{-3}$, due to the significant pile-up of gas in its Hill sphere. Conversely, for mass ratios below $q \leq 3 \times 10^{-4}$, which do not show dominant Hill sphere torques and do not reach as fast inspiral rates, one can safely estimate the average torque with analytical predictions for perturbers on fixed (circular) orbits and neglect any effects from the GW inspiral. We caution, however, that this only applies to the parameter space explored in this work, and disks with higher Mach numbers may lead to stronger deviations from analytical predictions.

Interestingly enough, our $q=3 \times 10^{-4}$ simulation in the $\alpha=0.03$ disc experiences a sign change in the torque as it approaches merger. In this case, gas torques would initially slow down the inspiral before accelerating it, and in principle the net effect of gas torques in the GW signal (discussed below in Section 5) may be diminished. We do not expect such an occurrence to be common, given that for the majority of our simulations, torques do not-on average-change sign. Furthermore the sign change occurs at migration rates near the ISCO (see Fig. 3), where as the EMRI spends most of its time (and thus is more likely to be observed) at larger separations where the torque remains positive. For reference, we note in the last column of Table 1 which simulations show significant deviations from steady-state torques prior to merger.

2 We note that the decreasing trend in the gas torque reported in Paper I was in fact a long-term transient due to the simulation having a closer outer boundary ( $r / r_{0} \leq 2.75$, rather than 6 ). Our slower inspiral runs confirm that the normalized torque on a $q=10^{-3}$ IMRI is indeed constant (albeit with oscillations) until reaching separations near $\sim 4 r_{\mathrm{S}}$, although this depends on the accretion rate. 

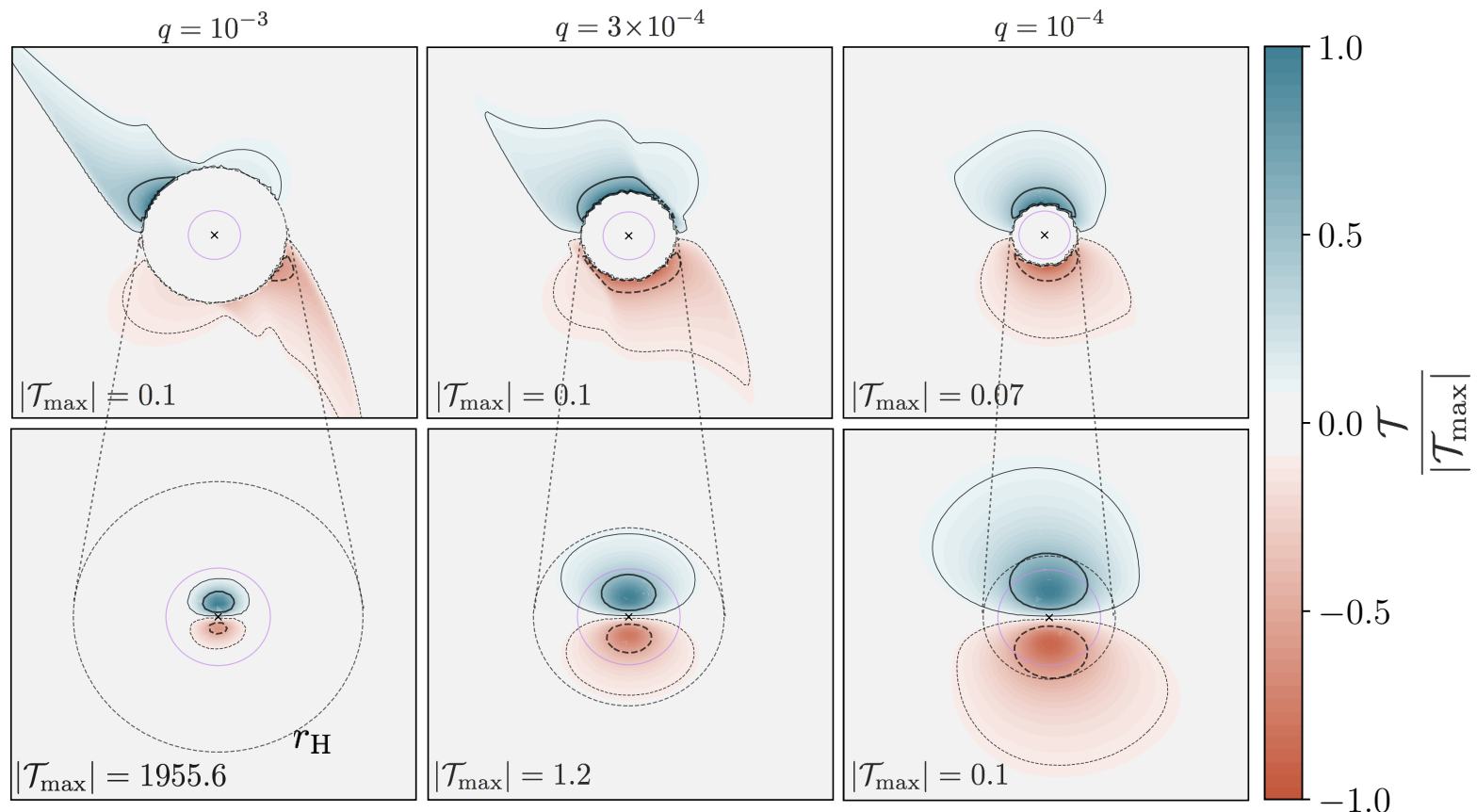

Figure 8. 2D contours of torque surface density $\left(\mathcal{T} \equiv \mathbf{g}_{\phi} \times \mathbf{r}\right.$, equivalently a torque per unit disc surface area) close to the satellite BH for discs with $\alpha=0.03$ and $\mathcal{M}=20$ and three different mass ratios. In the top panels we have excised the gas in the Hill radius ( $r_{\mathrm{H}}$; dashed circles) to highlight the gas morphology in streams nearby. The bottom panels show zoom-in views of the torque contributed by gas within the Hill sphere. All contours are normalized by the maximum $\mathcal{T}$, printed in each panel for reference. Note that gas pile-up for $q=10^{-3}$ is deep within the Hill radius and reaches significantly high densities. This results in much higher torque densities. The smoothing length of the gravitational potential is denoted with the solid purple circles.

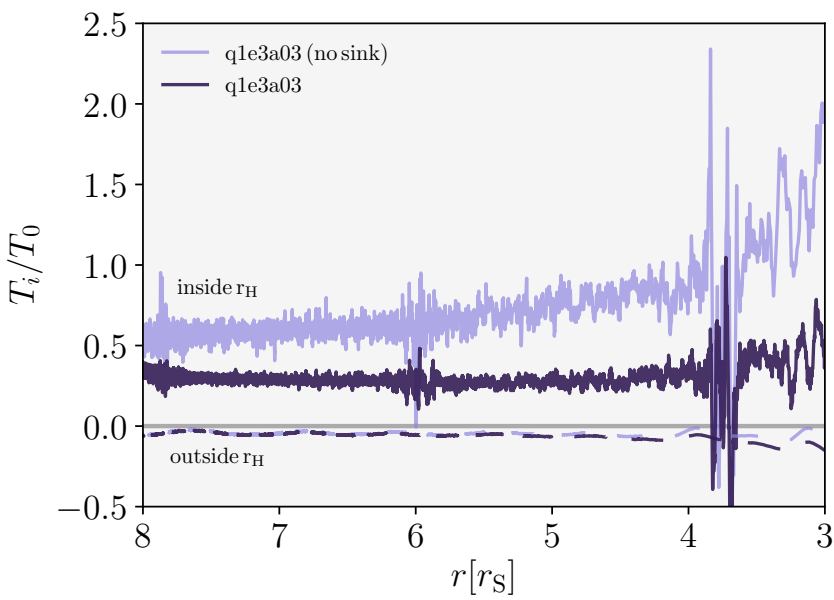

Figure 9. Torque on a $q=10^{-3}$ mass ratio inspiral divided into contributions from within and outside of the Hill radius, compared to the torques in a run with no accretion (i.e. with the sink turned off). The contributions from inside [outside] $r_{\mathrm{H}}$ are positive [negative]. Accretion reduces the gas density and damps the torque component inside the Hill radius, which otherwise would be affected by the increasing inspiral rate.

Finally, the inspiral has an impact on torque fluctuations. In particular for $q=10^{-3}$, for which gas pile-up on the $\mathrm{BH}$ is most significant, the Hill sphere torque shows an increase in fluctuation amplitude in the final $\sim 1000$ orbits. With a higher Mach number, these fluctuations are more extreme and arise at earlier times. In

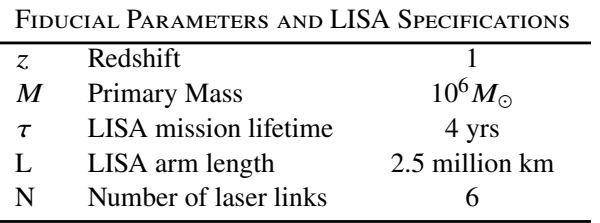

Table 2. LISA parameters are used when computing detectability.

particular the spikes in the Hill torque for $q=10^{-3}$ and $\mathcal{M}=20$ may signify an interesting dynamical interaction between the BH's orbit and the gas, given that they occur at the same radii regardless of viscosity or the $\mathrm{BH}$ sink rate. In simulations with varying boundaries $\left(0.4-0.5 \leq r / r_{0} \leq 3.0-6.0\right)$ or higher resolution (800, rather than 666 radial cells), we find that these spikes in the torque still occur at the same physical radii.

\section{SIGNIFICANCE FOR LISA INSPIRALS}

For the rest of the paper we take the results from our simulations, primarily the average torques measured in the $\alpha=0.03$ and $\alpha=0.1$ runs, and analyse their detectability in the GW signal. First we introduce some relevant quantities that describe a GW event in the LISA band, with the goal of computing the detectability of the imprint of the gas torques.

As illustrated in Figure 2, the inspiral rate for each mass ratio corresponds to a physical separation and gravitational wave frequency. Recall that the GW frequency is twice the orbital frequency for a binary on a circular orbit: $f=1 / \pi \sqrt{G M / r^{3}}$, and we have 
chosen these inspiral rates to correspond to IMRIs in the LISA frequency band.

The GW amplitude of a circular inspiral depends on the source distance (or redshift $z$ ), its frequency, and its chirp mass $\mathcal{M}_{\mathrm{c}}=$ $M_{1}^{3 / 5} M_{2}^{3 / 5} /\left(M_{1}+M_{2}\right)^{1 / 5}$. The sky- and polarization-averaged $\mathrm{GW}$ strain amplitude of a source at co-moving coordinate distance $r(z)$ is given by

$h=\frac{8 \pi^{2 / 3}}{10^{1 / 2}} \frac{G^{5 / 3} \mathcal{M}_{c}^{5 / 3}}{c^{4} r(z)} f_{r}^{2 / 3}$,

where $f$ is the observed GW frequency and $f_{r} \equiv f(1+z)$ is the GW frequency in the source's rest frame.

The characteristic strain $h_{c}$ of a source whose frequency evolves during a LISA observation of time $\tau$ (i.e. the LISA lifetime) is given by $h_{c}=h \sqrt{n}$, where $n \equiv f^{2} / \dot{f}$, which is a measure of the total number of cycles the source spends at each frequency (see Sesana et al. 2005 for a more detailed discussion). In Fig. 10 we plot the characteristic strain of IMRIs at each simulated mass ratio for two different possible observations of duration $\tau=4$ years, the currently planned nominal LISA mission lifetime (Amaro-Seoane 2018). We assume a fiducial primary mass $M_{1}=10^{6} M_{\odot}$ and place the source at redshift $z=1$. These parameters are listed in Table 2 . The dashed lines correspond to the final 4 years of a binary prior to reaching ISCO at $r_{\mathrm{ISCO}}=3 r_{\mathrm{S}}$, and the shorter, solid lines correspond to binaries observed for 4 years and ending up at $10 r_{\mathrm{S}}$ (in their rest frame).

The signal to noise ratio (SNR) of the event is a measure of its "loudness", or a way to characterise its detectability compared to the LISA noise. It is an integral of the strain amplitude over the noise, given in the stationary phase approximation by

$\rho^{2}=2 \times 4 \int_{f_{\min }}^{f_{\max }} d f \frac{|\tilde{h}(f)|^{2}}{S_{n}^{2}(f) f^{2}}$.

The pre-factor of two assumes the currently planned configuration of six links (effectively two independent interferometers), and $S_{n}(f)$ is the spectral density of LISA's noise per frequency bin, adopted from Klein et al. (2016), where we assume an arm length of 2.5 million $\mathrm{km}$ and include an estimate of confusion noise produced by foreground sources (galactic binaries).

For a fixed primary mass, binaries with lower mass ratios (smaller $\mathcal{M}_{\mathrm{c}}$ ) emit weaker gravitational waves, and thus span shorter frequency windows during a fixed observation time $\tau$. This reduces the total SNR as well as the chances of detecting a deviation in the signal.

\subsection{The imprints of gas on GWs}

\subsubsection{Phase drift}

Depending on the mass ratio and viscosity, gas torques either slow down or speed up the inspiral. For a gravitational wave event in the LISA data stream, this produces a phase drift in the waveform compared to that in vacuum, and a shift in the total phase accumulated during the event. If the accumulated phase (often defined in Fourier space) due to $\mathrm{GW}$ emission alone is $\Phi_{\mathrm{GW}}(f)$, the phase of an event experiencing gas torques will be $\Phi_{\mathrm{GW}}(f)+\delta \phi(f)$, i.e. the underlying vacuum signal plus some small deviation which can also depend on frequency. If the phase deviation $\delta \phi(f)$ is significant (and unique), the gas imprint is potentially distinguishable from the vacuum waveform (and from other deviating effects). This depends on (i) the strength of the gas torque compared to GWs, which changes with radius; (ii) the frequency window that is observed, and (iii) the

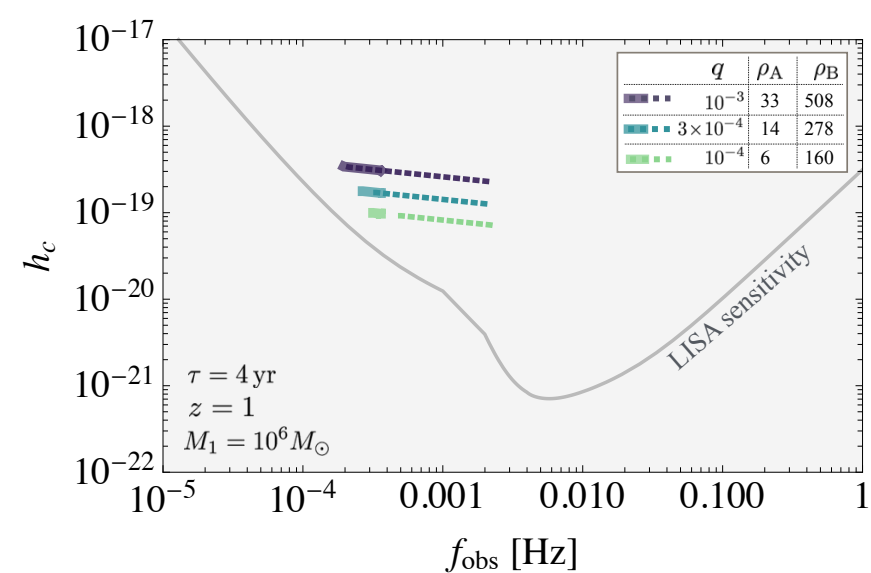

Figure 10. The characteristic strain as a function of observed frequency against the dimensionless LISA sensitivity. For each mass ratio, we show two possible observations of duration $\tau=4$ years, using our fiducial parameters $M_{1}=10^{6} M_{\odot}$ and $z=1$. Solid lines (observation 'A') correspond to an earlier evolutionary stage of a binary that reaches a rest-frame separation of $10 r_{\mathrm{S}}$. Dashed lines (observation 'B') cover the final coalescence of the binary, that reaches a rest-frame separation $r_{\mathrm{ISCO}}$. In the legend, we note the total SNR accumulated in each of these observations at early stages $\left(\rho_{\mathrm{A}}\right)$ and later stages $\left(\rho_{\mathrm{B}}\right)$, computed from Eq. 17 . The noise function is provided by Klein et al. 2016 .

signal to noise ratio (SNR) of the event itself. Thus $\delta \phi$ is not only a function of frequency, since torques evolve with radius, but also scales with the disc surface density as this determines the strength of the torque.

For calculating the phase shift induced by gas we take the same approach as in Paper I (Derdzinski et al. 2019), but implement the updated result for the gas torque and apply this to all simulated mass ratios. Recall that the total accumulated phase (in radians) of an event over a rest frame separation window is

$\Phi_{\mathrm{tot}}=-2 \pi \int_{r_{\mathrm{f}}}^{r_{\mathrm{i}}} \frac{f}{\dot{r}} d r$

where we assume the inspiral remains circular, and $\dot{r}$ is the inward radial acceleration. For an event in vacuum, this is purely driven by GWs and $\dot{r}=\dot{r}_{\mathrm{GW}}$. In a disk, gas torques also affect the orbital evolution and $\dot{r}=\dot{r}_{\mathrm{GW}}+\dot{r}_{\mathrm{gas}}$. Note that because our measured torques on average do not vary significantly throughout the inspiral, we assume that these components are not coupled. The phase shift of a gas-embedded event is then given by difference between the phase integral in vacuum and the observed accumulated phase: $\delta \phi=\left|\Phi_{\text {tot }}\left(\dot{r}_{\mathrm{GW}}\right)-\Phi_{\mathrm{tot}}\left(\dot{r}_{\mathrm{GW}}+\dot{r}_{\mathrm{gas}}\right)\right|$. Given that gas torques are much weaker than GWs $\left(\dot{r}_{\text {gas }} \ll \dot{r}_{\mathrm{GW}}\right.$, see Fig. 4$)$, we can simplify the latter integral and solve for the phase shift (or phase drift, as it accumulates) by

$\delta \phi=2 \pi \int_{r_{\mathrm{i}}}^{r_{\mathrm{f}}} \frac{f(r) \dot{r}_{\mathrm{gas}}}{\dot{r}_{\mathrm{GW}}^{2}}\left[1+O\left(\frac{\dot{r}_{\mathrm{gas}}}{\dot{r}_{\mathrm{GW}}}\right)^{2}\right] d r$,

where $\dot{r}_{\text {gas }}=2 T_{\text {gas }} M_{2}^{-1} r^{1 / 2}(G M)^{-1 / 2}$ is the change in separation due to gas torques. Note that $\dot{r}_{\text {gas }}$ can be a function of radius, as we define explicitly below, but that this function can vary for systems with different mass ratios or disc parameters.

As shown in our fiducial runs in Fig. 3, the magnitude of the gas 
torque when normalized by $T_{0}$ is approximately constant throughout the inspiral for all mass ratios. To compute the phase shift we use the average of these torques, calculated over the last 2000 orbits of the slower-inspiral simulations. We neglect the short time-scale oscillations in the torques, as well as the deviations from the average that occur at the highest inspiral rates. Given that normalised torques either stay constant or increase in absolute value with inspiral rate, this provides a lower limit on detectability.

If $T_{\mathrm{g}} / T_{0}$ is constant throughout the inspiral, then torques scale with the radial dependence of $T_{0}$, which is dependent on the initial disc density profile. We can define the average gas torque analytically as

$\left\langle T_{\text {gas }}\right\rangle=C_{\text {fit }} T_{0}(q, r, \mathcal{M}, \alpha, \Sigma(r))$,

where $C_{\text {fit }}$ is the (constant) average of the torque before it begins to deviate due to rapid inspiral. These fits are shown as horizontal dashed lines in Fig. 3 and also provided in Table 1.

The gas torque on the satellite $\mathrm{BH}$ can be expressed in terms of the rate of change of specific angular momentum $\dot{\ell}_{\text {gas }}=T_{\text {gas }} / M_{2}$, which relates to the rate of change of separation as $\dot{r}_{\text {gas }}(r)=$ $\frac{2}{\sqrt{G M_{1}}} r^{1 / 2} \dot{\ell}_{\text {gas }}(r)$. Plugging this expression into Eq. 19 allows us to solve for the shift in GW phase due to the gas torque on each binary, provided an observed frequency window (and corresponding range of separations). Note that in converting the torque from code units to physical units, these quantities must be scaled with our fiducial parameters by $T_{\text {gas }}=T_{\text {code }} \times G M_{1} \Sigma_{0} r_{0}$ and $\dot{\ell}_{\text {gas }}=T_{\text {code }} \times G \Sigma_{0} r_{0} / q$.

We integrate over the two different frequency windows for each mass ratio, defined by the 4 -yr observations shown in Fig. 10. Since our simulated inspiral does not cover the entire observed frequency range, we extrapolate the torque fit from Eq. 20 to the earlier stages, which implicitly (and reasonably) assumes that torques continue to scale with $T_{0}$ at earlier times.

In Fig. 11, we plot the total accumulated phase shift for each observation as a function of the disc surface density. $\delta \phi$ scales linearly with $\Sigma_{0}$, but accumulates to different values depending on the strength of the torque and the frequency window observed (both of which depend on $q$ ). For reference, we mark the surface densities in the $\alpha$ - and $\beta$-discs at $r_{0}=3 r_{\mathrm{S}}$ by vertical lines. In the low-density limit, gas torques only impart a phase shift of $\delta \phi \lesssim 10^{-3}$, which is likely undetectable. For higher densities $\left(\Sigma_{0} \gtrsim 10^{5} \mathrm{~g} \mathrm{~cm}^{-2}\right)$, the phase shift can exceed several radians.

\subsubsection{Detectability of waveform deviation}

The detectability of a deviation can be estimated by calculating the SNR of the difference between the two waveforms $(\delta \phi)$. Similarly to Eq. 17, we calculate the SNR of the deviation as

$\rho_{\delta \phi}^{2}=2 \times 4 \int_{f_{\min }}^{f_{\max }} d f \frac{|\delta \tilde{h}(f)|^{2}}{S_{n}^{2}(f) f^{2}}$,

where instead of the strain amplitude we integrate the strain deviation in Fourier space,

$|\delta \tilde{h}|^{2}=|\tilde{h}|^{2}\left(1-e^{i \delta \phi}\right)^{2}=2|\tilde{h}|^{2}(1-\cos (\delta \phi))$

This assumes the chirp is slow and that gas only imparts a difference in GW phase and not amplitude, also known as the stationary phase approximation (see Yunes et al. 2011 and Kocsis et al. 2011).

We show the accumulated SNR of the gas-induced deviation for the $\alpha=0.03$ runs in Fig. 12 as a function of disc surface density normalisation $\Sigma_{0}$. This allows us to assess, given a disc density, how distinguishable the phase-shifted waveform will be from the vacuum

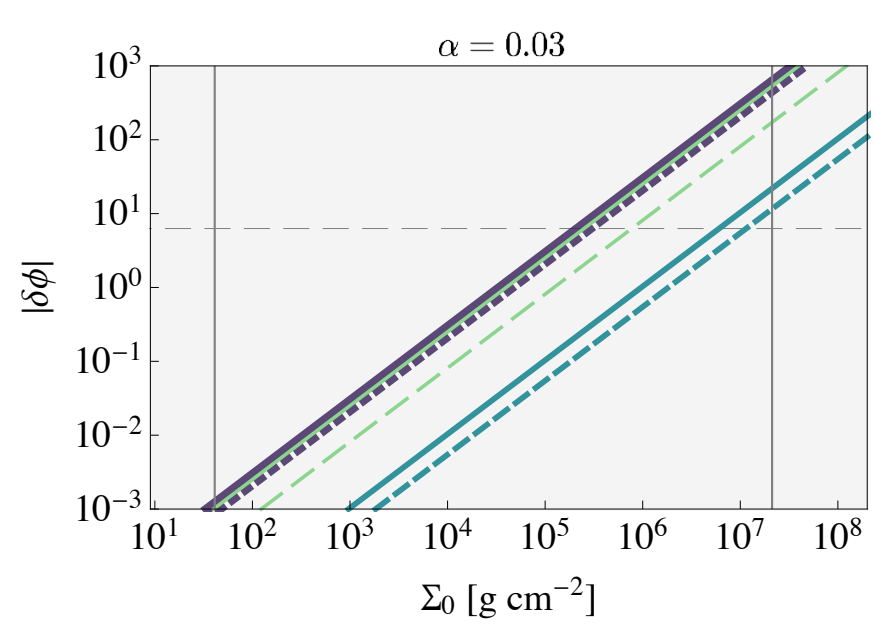

Figure 11. Absolute value of the total accumulated phase shift (Eq. 19) for each observation window depicted in Fig. 10, due to the average gas torque in the fiducial $\alpha=0.03$ runs. The colors and line types correspond to the same set of runs as in Figure 10, covering a pair of observational windows for three different mass ratios. Note that $\delta \phi$ can be negative or positive, depending on the sign of the torque. Vertical lines mark estimates for the disc surface density in the $\alpha$-disc and $\beta$-disc models at $r_{0}=3 r_{\mathrm{S}}$. The dashed horizontal line delineates when $\delta \phi=2 \pi$, above which the shift exceeds an entire period.

waveform. Just as the phase shift depends linearly on the surface density, $\rho_{\delta \phi}$ initially scales linearly with $\Sigma_{0}$. This can be interpreted from Eq. 22, where for small $\delta \phi$ the strain deviation becomes linear with the phase shift, as $|\delta \tilde{h}| \propto(1-\cos \delta \phi)^{1 / 2} \approx \delta \phi$. However, at high enough surface densities, where torques shift the phase by a whole period $(\delta \phi \gtrsim 2 \pi)$, this linear dependence disappears and the SNR saturates. This behavior is observed in binaries that are essentially monochromatic in frequency, an inevitable feature of circular, extreme mass ratio inspirals that coalesce very slowly. The exception is for binaries that are approaching merger, whose phase shift accumulates past $2 \pi$ as they sweep through higher frequencies. If an IMRI is embedded in a disc with surface densities reaching that of the $\beta$-disc model, and we observe the late stages of coalescence, its waveform may be significantly altered.

Ultimately the detectability of a deviation relies on the event accumulating substantial total SNR. Indeed, the observations for which a phase drift accumulates the highest $\rho_{\delta \phi}$ are those that accumulate highest total SNR, as indicated in Fig. 10. In Fig. 13, we show the SNR of the deviation divided by the total SNR of the event (a relative SNR, $\rho_{\delta \phi} / \rho$ ), as a function of disc surface density. This illustrates that the accumulated deviation for inspirals that are chirping faster (dashed lines) is weaker than for the inspirals that are observed at earlier times (solid lines). Nevertheless, we see in Fig. 12 these weaker deviations are more detectable due to the larger overall SNR. Figure 10 clearly illustrates the reason: these tighter binaries are observed at frequencies close to the minimum of LISA's sensitivity curve. Louder events - ideally intermediate mass ratios at low redshift - are the most promising candidates for detecting gas imprints.

Adopting a detectability threshold of $\rho_{\delta \phi} \gtrsim 8$, we conclude that the gas imprint is detectable for all simulated mass ratios if the disc density exceeds $\Sigma_{0} \gtrsim 10^{4-6} \mathrm{~g} \mathrm{~cm}^{-2}$. For an inspiral beginning at $10 r_{S}$, this threshold corresponds to a local disc mass of $M_{\mathrm{enc}} \sim$ 


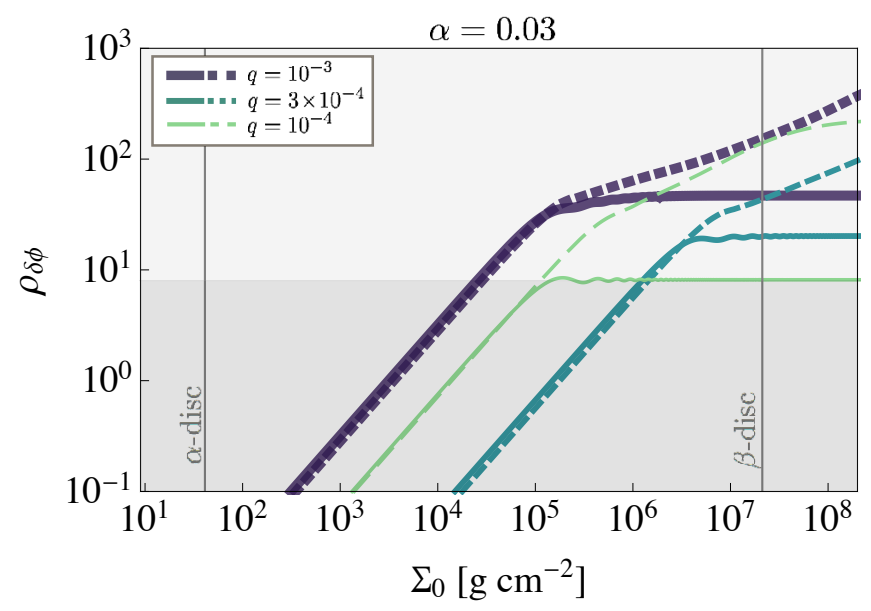

Figure 12. Accumulated SNR of the waveform deviation due to the average gas torque in our $\alpha=0.03$ runs, assuming binary parameters $M_{1}=10^{6} M_{\odot}$ and $z=1$ and $\tau=4$ years. Observational windows correspond to those depicted in Fig. 10. Observations of binaries at lower frequencies (solid lines) saturate once the phase shift reaches $\delta \phi \approx 2 \pi$. Binaries at later stages of coalescence continue to accumulate a larger phase shift due to the changing frequency, allowing higher values of disc density to yield large deviations. The shaded region corresponds to $\rho_{\delta \phi}<8$. The phase shift is most detectable for binaries that are chirping significantly in dense discs, although the exact threshold varies for each $q$.

$\pi r^{2} \Sigma_{0}=10^{-3}\left(\frac{r}{10 r_{S}\left(M_{1}\right)}\right)^{2}\left(\frac{\Sigma_{0}}{10^{5} \mathrm{gcm}^{-2}}\right) M_{\odot}$ within the vicinity of the secondary $\mathrm{BH}$. The surface density required for detectability depends on the strength of the torque, which varies for each value of the mass ratio. ${ }^{3}$ Given that the gas torque on the $q=3 \times 10^{-4}$ binary is an order of magnitude weaker than for the higher mass ratios, it requires a correspondingly higher $\Sigma_{0}$ for detectability. The detectability of gas for lower mass ratios at earlier stages suffers from weaker GW emission and more modest frequency evolution, and gas torques are less detectable even for the highest disc densities.

For higher viscosities, where torques are stronger for each mass ratio, the detectability of a deviation is improved (see Fig. 14), and an SNR deviation of $\rho_{\delta \phi} \gtrsim 8$ can be reached with lower disc densities, $\Sigma_{0} \sim 10^{4} \mathrm{~g} \mathrm{~cm}^{-2}$.

One may wonder how our choice of primary mass affects the detectability of the gas imprints. In principle, IMRIs can occur for more or less massive primary MBHs while still emitting GWs within the LISA frequency band. We demonstrate the effect of our choice of primary mass $M_{1}$ for a fixed mass ratio $q=10^{-3}$ in Fig. 15 . Higher mass binaries emit louder gravitational waves, but they merge at lower frequencies due to their increasingly large ISCO $\left(r_{\mathrm{S}} \propto M\right)$. Taking the mass ratio $q=10^{-3}$, we plot the strain and corresponding detectability of the phase shift, adopting the dimensionless gas torque from our fiducial run (q1e3a03, where $\left\langle T_{\text {gas }}\right\rangle / T_{0}=0.21$ ). We fix each observation window to the binary reaching $3 r_{\mathrm{S}}$ in a 4 year observation. In this case the lower-mass binary can exhibit more detectable gas torques, given that the coalescence occurs at

3 Compared to Paper I, the phase shift for $q=10^{-3}$ requires a slightly higher $\Sigma_{0}$ for detectability. This is primarily because we fit $T_{\mathrm{g}} / T_{0}$ to a constant (rather than the decreasing polynomial shown in Paper I) which results in a weaker estimate for the torque strength. Additionally, we are using a more conservative observation time of 4 years (rather than 5).

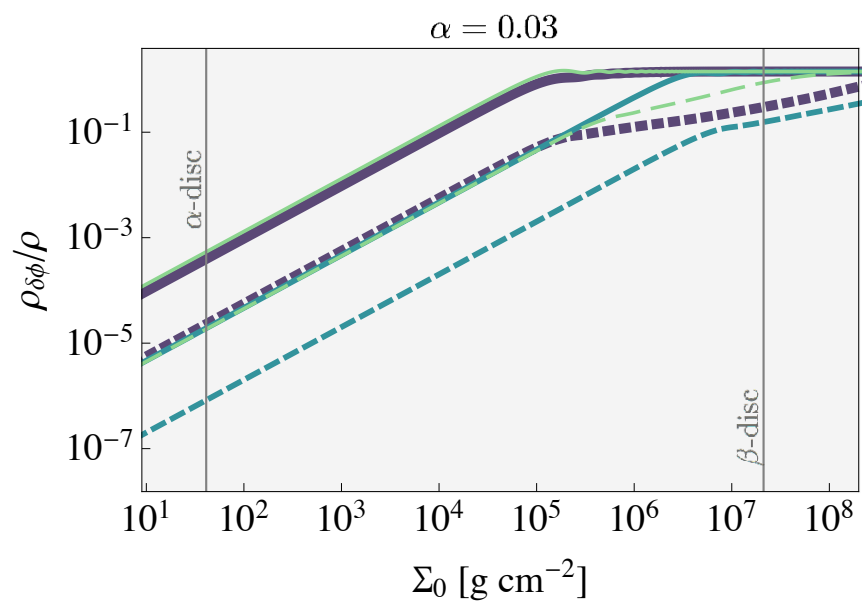

Figure 13. Relative SNR (i.e. the SNR of the deviation divided by the total SNR of the event) for the $\alpha=0.03$ runs, with the same color key as in Fig. 10. This shows that a faster chirp signal is relatively less affected by the gas torques, but the gas imprint is still more detectable because of the higher total SNR of the event (seen in Fig. 10).

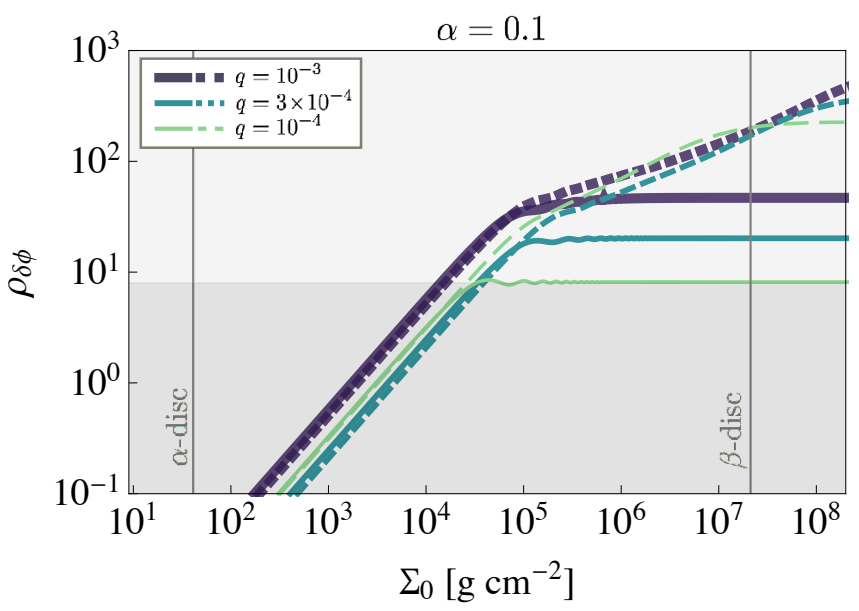

Figure 14. Accumulated SNR of the deviation due to average torques in the $\alpha=0.1$ runs, shaded up to our detectability threshold of $\rho_{\delta \phi} \geq 8$. Higher viscosity generally produces stronger torques; hence the imprint is detectable at lower disc densities.

frequencies where LISA is most sensitive. However, the overall SNR of the event (and consequently the SNR of the deviation) will depend on the range of observed frequency of the binary and its relation to the peak sensitivity-notice that the coalescence of the $10^{6} M_{\odot}$ IMRI attains the highest total SNR (dashed purple line, $\rho_{\mathrm{B}}$ ), while the $10^{5} M_{\odot}$ merger occurs at higher frequencies, reducing the total SNR (dashed orange line, $\rho_{\mathrm{B}}$ ). Additionally, lower-mass binaries span a larger frequency range in a fixed observation time, simply because at fixed $r / r_{\mathrm{S}}$ the frequency evolution rate $\dot{f}$ scales more steeply with frequency than chirp mass $\left(\dot{f} \propto M_{c}^{5 / 3} f^{11 / 3}\right)$. In summary, the detectability of the gas deviation is tied to the stage of the coalescence we observe - binaries that are chirping in the cusp of 

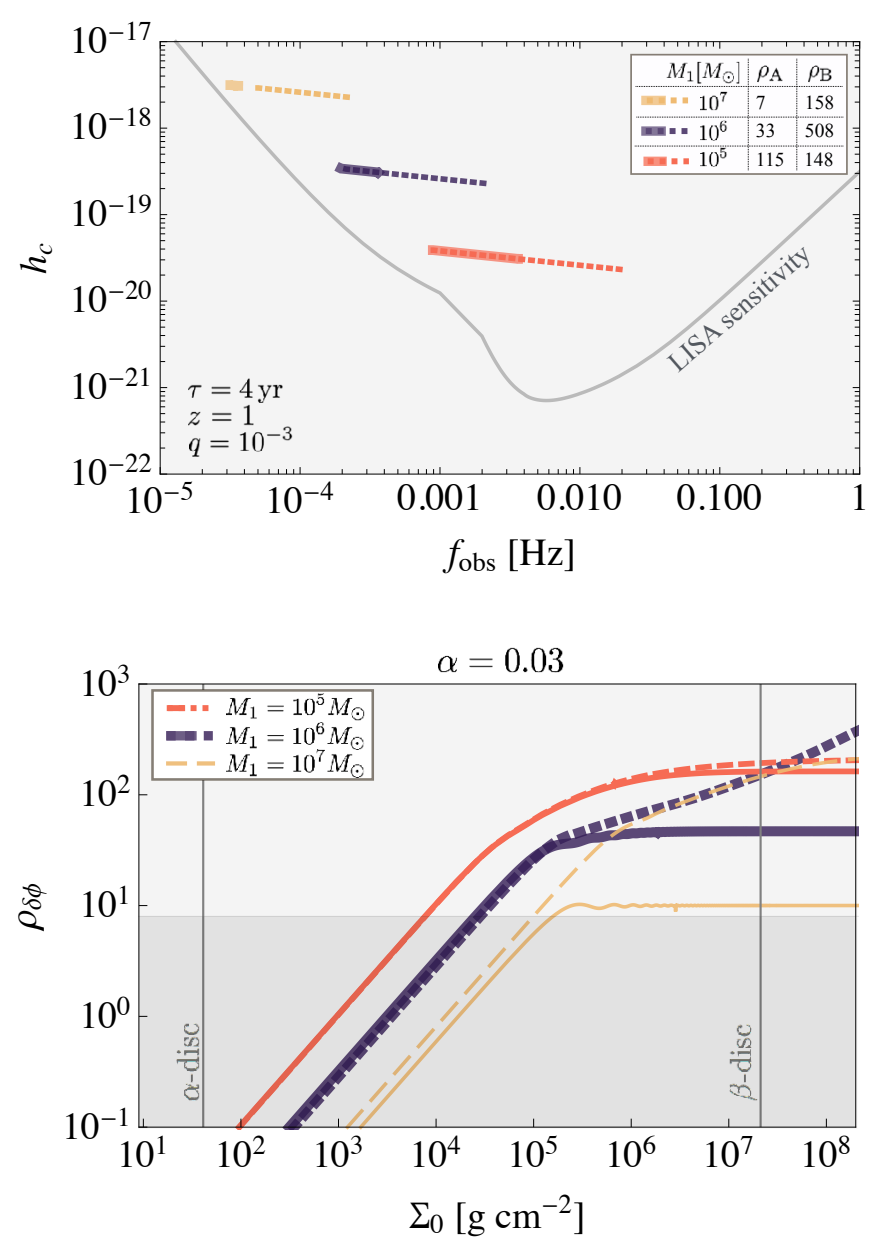

Figure 15. Top panel: Characteristic strain $v s$. observed frequency for 4year observations of $q=10^{-3}$ binaries at $z=1$, varying the primary mass $M_{1}$ from $10^{5} M_{\odot}$ to $10^{7} M_{\odot}$. Solid lines correspond to early stages of evolution where the binary reaches a rest-frame separation of $10 r_{\mathrm{S}}$. Dashed lines correspond to observing the final coalescence, ending when the binary merges at $r_{\text {ISCO }}=3 r_{\mathrm{S}}$. In the legend we provide the accumulated SNR for the early ('A') and late ('B') observations, respectively. For lower binary mass, the merger occurs at a smaller $r_{\text {ISCO }}$ and correspondingly higher frequencies. This affects the detectability of the event. Bottom panel: SNR of gas deviation as a function of disc surface density given the observation windows depicted above, and using the average dimensionless gas torque from the $q=10^{-3}$ and $\alpha=0.03$ runs. A binary with $M_{1}=10^{5} M_{\odot}$ accumulates a stronger waveform deviation as it spans a larger frequency range. Note that the vertical line for the $\Sigma_{\beta}$ estimate corresponds to a disc around an $M_{1}=10^{6} M_{\odot} \mathrm{BH}$; see Eq. 13 for the weak scaling of the disc surface densities with BH mass.

LISA's sensitivity are the most promising candidates for extracting a phase shift.

\subsection{Uniqueness and Degeneracies}

It is important to consider whether the deviations we find in the waveform are degenerate with changes in the chirp mass or other system parameters, and/or with other possible environmental effects, and if such degeneracies may hinder parameter estimation or chances of detection.
A critical feature for distinguishing between various environmental effects and system parameters is the respective scaling of each effect with binary separation or GW frequency. For example, some proposed modifications to general relativity predict waveform deviations that would increase as the EMRI coalesces, scaling with a predictable power of frequency ${ }^{4}$ (Yunes 2009). In principle a deviation to the waveform could be interpreted as a binary with different system parameters that also determine the frequency evolution, such as chirp mass $M_{c}$. This can be distinguished by how various parameters affect the waveform as the frequency evolves. This dependence is often quoted in Fourier space, where deviations can be compared to Post-Newtonian (PN) terms in the Fourier phase. The scaling of changes in parameters with frequency can then be compared amongst system parameters, external effects, or modifications of general relativity.

Neglecting short-timescale fluctuations, our simulated gas torque scales with the analytical estimate $T_{0}$, which is a function of the disc density profile. Given our disc model, $T_{\text {gas }} \propto$ $r^{4} \Omega^{2} \Sigma(r) \propto r^{1 / 2}$. The viscous torque also carries the same scaling with radius, given by $T_{v} \propto r^{2} \Omega v(r) \Sigma(r) \propto r^{1 / 2}$. This predicts that gas torques get progressively weaker as the binary coalesces: if $f_{\mathrm{GW}} \propto r^{-3 / 2}$, then the gas torque initially scales with GW frequency as $T_{\mathrm{gas}} \propto f_{\mathrm{GW}}^{-1 / 3}$, and the integral for the deviation scales as $\delta \phi \propto f^{-13 / 3}$. This frequency-scaling can be compared to the phasing function for a circular inspiral around a non-spinning $\mathrm{BH}$, where $1.5 \mathrm{PN}$ terms scale with frequency as $\phi \propto A f^{2 / 3}+B f$ (where $A$ and $B$ are constants that depend on system parameters, see a more detailed description in Will \& Yunes 2004). In this case, gas torques scale uniquely with frequency, suggesting that the impact of the gas torque is not degenerate with system parameters or GR corrections. However, confirming a phase drift to be of gas-disc origin, rather than other possible environmental effects, may only be possible for events that span a sufficiently large range of frequencies.

These considerations exclude evolutions in the dimensionless torque that can occur at the fastest inspiral rates, particularly for low-viscosity discs, or if the $\mathrm{BH}$ migrates through a disc where parameters (e.g. $\alpha, \mathcal{M})$ vary with radius. It also neglects the oscillations that occur in the torque which we see for all simulated inspiral rates. If measurable, these time-variable fluctuations could be a smoking-gun signature of disc response to an embedded IMRI. Ultimately the frequency dependence of the effect varies with disc physics, and changes in $\alpha, \mathcal{M}$, or accretion efficiency will lead to different scalings.

\subsection{Caveats}

This study provides a crucial first step in determining how gas torques respond to a GW-driven inspiral, but much work must be done to make more accurate observational predictions. These simulations are numerically challenging in that they require a large boundary (to avoid transient effects) and high spatial resolution (to resolve the gas in the immediate vicinity of the satellite $\mathrm{BH}$ ), they must model the global disc (the small-scale gas dynamics near the satellite depends on the global disc), and they must be evolved for several thousand dynamical times for each set of parameters (to avoid transient behaviour reflecting the initial conditions). In the

\footnotetext{
4 However, one would expect effects from modified gravity to be present in all E/IMRIs within a certain frequency range, while gas should only affect a subset of the events.
} 
interest of computational efficiency, we have neglected several physical processes that are important for modeling a realistic system. We summarize some of our limitations here.

Our simulations do not resolve 3-dimensional gas morphology which may be important for resolving accurate torques (Tanigawa et al. 2012; Szulágyi et al. 2014; Morbidelli et al. 2014), although in some parameter regimes $2 \mathrm{D}$ simulations are sufficiently accurate (e.g. see Lega et al. 2015; Uribe et al. 2011). In particular, the asymmetric density distribution that determines the gravitational torques is in some cases concentrated near or even inside the smoothing length. The dense pile-up is only a factor of $\sim$ two inside the smoothing length in the most extreme case (i.e. for $q=10^{-3}$; see Fig. 8). It is useful to keep in mind that unlike in N-body or smoothed particle hydrodynamics simulations, where smoothing is done purely for numerical stability, the smoothing we employ here is physically motivated: the smoothing of the potential mimics a vertical averaging of the gravitational forces. Nevertheless, the small-scale density distribution may be impacted by our choice of smoothing prescription, and 3-dimensional simulations will be necessary to fully understand how this morphology arises and if this gas distribution and the resulting torques are modified in 3D.

Our disc model is isothermal and does not include radiative cooling, heating, nor more sophisticated physics such as magnetohydrodynamics or radiation pressure, all of which may alter the gas dynamics near the BH. Future work must consider how gas morphology near the $\mathrm{BH}$ is affected by accretion rate and feedback from the BH itself, which may heat the gas in its vicinity and dampen the torque (Szulágyi et al. 2016). While feedback tends to reduce the gas density in the vicinity of the migrator, it can do so assymetrically, producing an additional 'heating torque' (as discussed in Hankla et al. 2020).

The choice of accretion prescription and sink timescale of the embedded BH should also be considered carefully. Our estimate assumes that accretion occurs on the viscous timescale via a thin disc around the $\mathrm{BH}$. However, given that the specific angular momentum of the gas with respect to the gap-opening perturber is low (and the resulting accretion torque is negligible, as discussed in Paper I), perhaps a quasi-spherical accretion prescription (i.e., Bondi accretion; Edgar 2004) would be more appropriate. The possibility for super-Eddington accretion rates should be considered, which can result in feedback that further affects the orbital properties of the $\mathrm{BH}$ (Gruzinov et al. 2020). We expect that the inclusion of such affects will affect the precise value of the torque and its variability, particularly for more massive IMRIs with $q \sim 10^{-3}$ which experience the strongest nonlinear effects and more significant accretion/feedback. Nevertheless, we do not expect these effects to significantly change our detectability results of gas imprints, as our estimates utilize the time-averaged torques. Rather more sophisticated treatments may change predictions of the precise signature in the waveform - i.e. the sign, strength, and frequency-dependence of the phase drift, ad any effects that lead to a strong increase or reduction in torque strength (such as significant feedback), will alter the specific disc density constraints for detectability.

Finally, our simulations are purely Newtonian, neglecting any relativistic effects which can alter gas dynamics in the inner regions of the accretion disc closer to the primary BH. However, we do not expect the inclusion of relativistic effects to substantially affect the detectability of the gas imprint, since the phase shift is mostly accumulated at larger separations. We assume the binary inspiral remains circular, when in fact gas discs may excite non-negligible eccentricity in the orbit (e.g. Goldreich \& Sari 2003; D’Angelo et al. 2006), and this eccentricity may also produce additional modulations in the torque. Additionally, our estimates of binary evolution may be slightly inaccurate due to our use of the Peters (1964) quadrupole formula for the inspiral rate, which is lacking PN terms that become important near the ISCO (Zwick et al. 2019).

\section{DISCUSSION}

In the present work we analyse torque evolution during GW-driven inspirals in the intermediate mass ratio regime. These sources, while their rates are less certain, provide the tantalizing possibility of probing nonlinear binary+gas-disc physics. At lower mass ratios, they evolve more slowly and quietly than near equal-mass $\mathrm{MBH}$ mergers, yet they maintain the ability to accumulate significant SNR.

In all cases, we observe short time-scale modulations in the torque throughout the inspiral. For most cases, the strength of the torque is on the order of simple analytical predictions (some fraction of $T_{0}$ ), but the precise value and the sign of the torque changes nontrivially with $q$ and $\alpha$. For our highest simulated mass ratio $q=10^{-3}$, where gas pile-up on the $\mathrm{BH}$ is significant, torques are positive (outward), noisy, and show a distinct increase in variability at the fastest inspiral rates. The strength and evolution of the torque in this case is sensitive to accretion efficiency: less efficient accretion can lead to an increasingly positive torque as the binary coalesces. This effect may be amplified in more massive IMRIs, but this must be confirmed with future simulations. For lower- $q$ inspirals, we find that torques are smoother and become more negative at fast inspiral rates, depending on the viscosity. Overall, IMRIs with different system parameters can experience different torque evolution.

Unsurprisingly, the dependence of torque with inspiral rate itself depends on disc parameters, namely $\mathcal{M}$ and $\alpha$. We find that inspirals in low $\alpha$ discs show deviations in the torque (compared to a constant dimensionless value) at earlier times. We interpret this as the disc's inability to respond to the satellite BH's increasing inspiral speed. Given the current understanding of viscosity in AGN discs (from estimates of $\alpha$ in MHD simulations), such low viscosities are unlikely. In the case of higher $\alpha$, torques may hold a relatively steady dependence on radius that scales with the viscous torque.

We find that fluctuations in the torque are highly dependent on the Mach number, or disc temperature. Hotter (low $\mathcal{M}$ ) discs produce smoother, "well-behaved" torques, while thinner, colder discs exert torques that are stronger (scaling on average with the $T_{0}$ prediction) and more variable. Our simulations of a $\mathcal{M}=30$ disc yields a torque with strong variations that oscillate between positive and negative values. This suggests that IMRIs in thin, supersonic discs may experience the most dynamic gas effects.

The variability in torques throughout the inspiral may be attributed to interesting gas dynamics that warrants further investigation. For example, our $q=10^{-3}$ simulations show peaks in the noise amplitude at $r \approx 8,6$ and $4 r_{\mathrm{S}}$ (most clearly seen in Fig. 9). These peaks occur at the same physical radii regardless of the simulation resolution, boundary location, or sink prescription. This raises the possibility that torques may show coherence with binary separation. We defer this analysis, as well as a closer look at the underlying cause of torque variability, to future work.

Assuming a gas-embedded IMRI event has sufficiently high SNR and spans a large enough frequency range that a frequencydependent waveform deviation $\delta \phi(f)$ can be measured, it will not only confirm the deviation to be of gas origin (at the least ruling out other possibilities), it can also provide an invaluable measure of disc properties as a function of radius. While a measure of ac- 
cumulated phase shift can place a constraint on the disc density $\Sigma_{0}$, in the most optimistic case a phase drift $\delta \phi(f)$ could reveal how the surface density changes with radius $\Sigma(r)$, which is a distinguishing factor amongst several accretion disc models. Understanding the complex physical processes at play in the inner regions of AGN accretion discs is an active field of research. Recent radiation, magneto-hydrodynamic simulations by Jiang et al. (2019) predict that inner disc regions may have lower densities than predicted by the $\beta$-disc model, although the densities should increase with accretion rate and may change with central MBH mass.

We note that these results are all subject to the limitations of our simplistic disc model, where the Mach number and aspect ratio do not vary with radius. This approach allows us to investigate whether changes in the torque are truly due to the GW inspiral, and not due to encounters with varying disc dynamics. Previous works suggest that torques in response to an artificially imposed migration may change sign at fast migration rates (Duffell et al. 2014), but those simulations utilise a disc model with constant surface density, implying a radially dependent aspect ratio. Comparison between these types of studies highlights the importance of considering how different disc models may affect an inspiraling BH. On this basis, IMRIs embedded in more physically-motivated discs - in which the Mach number and disc structure changes with radius - may show more extreme changes in the torque, although this remains to be confirmed with more sophisticated models.

The presence or absence of a phase drift should be considered in conjunction with other characteristics of the source, particularly any tell-tale signs of gas discs. A likely signature of a gas-embedded E/IMRI would be the combination of a phase drift with low eccentricity: gas-embedded E/IMRIs should be distinctly less eccentric than those expected to occur in dry galactic nuclei. They may be close to circular, but with mild gas-driven eccentricity $(e \lesssim 0.2$, Ragusa et al. 2018, D'Orazio et al., in prep, Zrake et al. in prep) or eccentricity induced by other embedded perturbers. Additionally, if the accretion disc is aligned with the spin of the central MBH (this may only be true in some cases to varying degrees, see Volonteri et al. (2013) and references therein), the spins of the binary components may be closely aligned. However, this will depend on the history and nuances of accretion onto the disc-born $\mathrm{BH}$, for which there are many uncertainties.

Our estimates of detectability of the deviation implicitly rely on the assumption that we have a waveform catalog of all possible I/EMRIs. Currently, the catalog of intermediate mass ratio waveforms is incomplete (Mandel \& Gair 2009). A lack of available waveforms will affect the accuracy with which we can extract binary parameters from the signal, let alone detecting subtle environmental deviations (Cutler \& Vallisneri 2007). To further complicate the picture, the range of possible AGN environments means that deviations may vary from system to system. In terms of data analysis, we suggest methods that search for generic deviations in a waveform, which can be informed by studies such as that presented here. In the optimistic case, one hopes that gas imprints can be traced back to constraints on the source environment. However, challenges will arise with circular, gas-embedded I/EMRIs that are in early stages of evolution, as these will arise in GW data as quasi-stationary sources. In these cases, assessing degeneracies, or disentangling the phase-shifted waveform between system parameters and various environmental effects, will not be possible if one cannot measure $\dot{f}$ of the source within a limited observation time.

Gas also provides the opportunity for electromagnetic emission that may coincide with the GW event. We do not address this issue here, but remind the reader that combining a phase shift with any associated EM signatures would be invaluable for confirming the presence of circumbinary gas and for learning about AGN discs. For example, the coalescence of a gap-opening secondary may accompany a change in AGN continuum that correlates with the mass ratio (e.g. Gültekin \& Miller 2012). Variable signatures in the broad component of $\mathrm{Fe} \mathrm{K} \alpha$ line emission, believed to be associated with the inner disk structure, may also indicate the presence of a gap-opening IMRI via periodic oscillations (McKernan et al. 2013). Such signatures would be detectable with the upcoming X-ray observatory Athena (Barret et al. 2020), and a powerful probe of inner disk morphology if observed concurrently with LISA (McGee et al. 2020). These events may also be correlated with the fading observed in changing-look quasars, where the characteristic timescales suggest the change in emission is due to an abrupt change in the structure of the innermost accretion disc (Stern et al. 2018). Such changes could by driven by thermal or magnetic instabilities and possibly triggered by embedded perturbers.

\section{CONCLUSIONS}

In this paper, we analyse the gas torques on an intermediate mass ratio binary inspiral embedded in an accretion disc. We present a suite of simulations of IMRIs embedded in two-dimensional, nearKeplerian, isothermal accretion discs, where the satellite BH is modeled as a smoothed point-mass with a sink prescription. We analyse the torque exerted by the gas on the inspiraling $\mathrm{BH}$ for a range of mass ratios $\left(10^{-4}<q<10^{-3}\right)$, disc viscosities $(0.01<\alpha<0.1)$, and Mach numbers $(10<\mathcal{M}<30)$. We also consider binaries at different states of the inspiral, and with and without allowing the satellite $\mathrm{BH}$ to accrete. Here we summarize our conclusions:

- As in similar numerical studies, we find that torques in the intermediate mass ratio, gap-opening regime have a nonlinear scaling with disc properties. Torques either slow down or speed up the inspiral; their strength is some fraction $(\sim 1 \%-120 \%)$ of the Type I torque $T_{0}$ (Tanaka et al. 2002), but these values are sensitive to small changes in $q, \alpha$, and $\mathcal{M}$.

- During the inspiral, the torques exerted by the gas on the satellite $\mathrm{BH}$ show weak fluctuations, but the average strength of the torque ( $T_{g}$ normalised by $T_{0}$ ) remains constant for the majority of inspiral rates in the LISA band. For the fastest inspiral rates, particularly for $q=10^{-3}$ approaching the ISCO, torques exhibit an increase in variability originating from the gas flow within the BH's Hill radius.

- We scale our simulation setup to a fiducial binary with primary mass $M=10^{6} M_{\odot}$ at $z=1$ in order to compute the detectability of gas-induced deviations in the GW waveform. Using the average of $T_{\text {gas }}$ for each mass ratio, we compute the accumulated phase shift and the corresponding SNR of the deviation as a function of disc density normalisation. We find that the phase shift is detectable (with relative $\operatorname{SNR} \rho_{\delta \phi}>8$ ) when the source is embedded in a disc with surface density $\Sigma_{0} \gtrsim 10^{4-6} \mathrm{~g} \mathrm{~cm}^{-2}$ (or a local disc mass $M_{\text {enc }} \sim 10^{-3} M_{\odot}\left(\Sigma_{0} / 10^{5} \mathrm{~g} \mathrm{~cm}^{-2}\right)$ ), depending on the mass ratio and disc viscosity. Detectability is maximized for the loudest events that are chirping significantly throughout a LISA observation, and coalesce at frequencies near $\sim 5 \times 10^{-3} \mathrm{~Hz}$ where LISA is most sensitive.

- This work is an important step towards understanding the scope of environmental impact on LISA sources due to circumbinary gas. Ultimately the strength, direction, and evolution of the torque exerted on a gas-embedded IMRI is dependent on the mass ratio and 
disc parameters, and the resulting waveform deviations can manifest in a variety of ways. A measure of a phase drift can provide a constraint on the disc surface density or disc structure, provided we have the tools to extract a variable deviation from the GW signal.

\section{Acknowledgements}

The authors thank the anonymous referee for insightful comments. AD acknowledges support by the National Science Foundation (NSF) Graduate Research Fellowship under Grant DGE 1644869. DJD acknowledges support from the Institute for Theory and Computation Fellowship. ZH acknowledges support from NSF grant 1715661 and NASA grants NNX17AL82G and 80NSSC19K0149. Computational resources were provided by the NASA High-End Computing (HEC) Program through the NASA Advanced Supercomputing (NAS) Division at Ames Research Center. We acknowledge the use of the following software: DISCO (Duffell 2016), Matplotlib (Hunter 2007), NumPy (van der Walt et al. 2011), Mathematica 12.0 (Inc. 2019).

\section{Data availability}

The simulation data that support the finding of this study are available from corresponding author $\mathrm{AD}$, upon reasonable request.

\section{REFERENCES}

Amaro-Seoane P., 2018, Phys. Rev. D, 98, 063018

Amaro-Seoane P., et al., 2017, preprint, (arXiv: 1702.00786)

Antoni A., MacLeod M., Ramirez-Ruiz E., 2019, ApJ, 884, 22

Armitage P. J., 2007, arXiv e-prints, pp astro-ph/0701485

Artymowicz P., Lin D. N. C., Wampler E. J., 1993, ApJ, 409, 592

Barausse E., Cardoso V., Pani P., 2014, Phys. Rev. D, 89, 104059

Barausse E., Cardoso V., Pani P., 2015, in Journal of Physics Conference Series. p. 012044 (arXiv:1404.7140), doi:10.1088/17426596/610/1/012044

Barret D., Decourchelle A., Fabian A., Guainazzi M., Nandra K., Smith R., den Herder J.-W., 2020, Astronomische Nachrichten, 341, 224

Bartos I., Kocsis B., Haiman Z., Márka S., 2017, ApJ, 835, 165

Baruteau C., Masset F., 2013, Recent Developments in Planet Migration Theory. p. 201, doi:10.1007/978-3-642-32961-6_6

Bellovary J. M., Mac Low M.-M., McKernan B., Ford K. E. S., 2016, ApJ, 819, L17

Chen X., Shen Z.-F., 2019, e-print arXiv:1906.11055, p. arXiv:1906.11055

Crida A., Baruteau C., Kley W., Masset F., 2009, A\&A, 502, 679

Cutler C., Vallisneri M., 2007, Phys. Rev. D, 76, 104018

D'Angelo G., Lubow S. H., 2010, ApJ, 724, 730

D'Angelo G., Lubow S. H., Bate M. R., 2006, ApJ, 652, 1698

D’Orazio D. J., Haiman Z., MacFadyen A., 2013, MNRAS, 436, 2997

D'Orazio D. J., Haiman Z., Duffell P., MacFadyen A., Farris B., 2016, MNRAS, 459, 2379

Dempsey A. M., Lee W.-K., Lithwick Y., 2020, ApJ, 891, 108

Derdzinski A. M., D’Orazio D., Duffell P., Haiman Z., MacFadyen A., 2019, MNRAS, 486, 2754

Dittmann A. J., Miller M. C., 2020, MNRAS, 493, 3732

Dong R., Rafikov R. R., Stone J. M., Petrovich C., 2011, ApJ, 741, 56

Duffell P. C., 2015, ApJ, 806, 182

Duffell P. C., 2016, ApJS, 226, 2

Duffell P. C., MacFadyen A. I., 2012, ApJ, 755, 7

Duffell P. C., Haiman Z., MacFadyen A. I., D’Orazio D. J., Farris B. D., 2014, ApJ, 792, L10

Duffell P. C., D’Orazio D., Derdzinski A., Haiman Z., MacFadyen A., Rosen A. L., Zrake J., 2020, ApJ, 901, 25

Edgar R., 2004, New Astron. Rev., 48, 843
Edgar R. G., 2007, ApJ, 663, 1325

Fabj G., Nasim S. S., Caban F., Ford K. E. S., McKernan B., Bellovary J. M., 2020, MNRAS, 499, 2608

Gayathri V., Bartos I., Haiman Z., Klimenko S., Kocsis B., Márka S., Yang Y., 2020, ApJ, 890, L20

Goldreich P., Sari R., 2003, ApJ, 585, 1024

Goldreich P., Tremaine S., 1980, ApJ, 241, 425

Goodman J., Tan J. C., 2004, ApJ, 608, 108

Gröbner M., Ishibashi W., Tiwari S., Haney M., Jetzer P., 2020, A\&A, 638, A119

Gruzinov A., Levin Y., Matzner C. D., 2020, MNRAS, 492, 2755

Gültekin K., Miller J. M., 2012, ApJ, 761, 90

Haiman Z., Kocsis B., Menou K., 2009, ApJ, 700, 1952

Hankla A., Jiang Y.-F., Armitage P., 2020, arXiv e-prints, p. arXiv:2005.03785

Hunter J. D., 2007, Computing in Science \& Engineering, 9, 90

Inc. W. R., 2019, Mathematica, Version 12.0, https://www. wolfram. $\mathrm{com} / \mathrm{mathematica}$

Jiang Y.-F., Blaes O., Stone J. M., Davis S. W., 2019, ApJ, 885, 144

Just A., Yurin D., Makukov M., Berczik P., Omarov C., Spurzem R., Vilkoviskij E. Y., 2012, ApJ, 758, 51

Kanagawa K. D., Tanaka H., Szuszkiewicz E., 2018, ApJ, 861, 140

Kennedy G. F., Meiron Y., Shukirgaliyev B., Panamarev T., Berczik P., Just A., Spurzem R., 2016, MNRAS, 460, 240

King A. R., Pringle J. E., Livio M., 2007, MNRAS, 376, 1740

Klein A., et al., 2016, Phys. Rev. D, 93, 024003

Kley W., Nelson R. P., 2012, ARA\&A, 50, 211

Kocsis B., Yunes N., Loeb A., 2011, Phys. Rev. D, 84, 024032

Krolik J. H., 1999, Active galactic nuclei : from the central black hole to the galactic environment

Lega E., Morbidelli A., Bitsch B., Crida A., Szulágyi J., 2015, MNRAS, 452, 1717

Levin Y., 2007, MNRAS, 374, 515

MacLeod M., Lin D. N. C., 2020, ApJ, 889, 94

Mandel I., Gair J. R., 2009, Classical and Quantum Gravity, 26, 094036

Masset F. S., D’Angelo G., Kley W., 2006, ApJ, 652, 730

Mayer L., 2013, Classical and Quantum Gravity, 30, 244008

McGee S., Sesana A., Vecchio A., 2020, Nature Astronomy, 4, 26

McKernan B., Ford K. E. S., Lyra W., Perets H. B., 2012, MNRAS, 425, 460

McKernan B., Ford K. E. S., Kocsis B., Haiman Z., 2013, MNRAS, 432, 1468

McKernan B., Ford K. E. S., Kocsis B., Lyra W., Winter L. M., 2014, MNRAS, 441, 900

McKernan B., et al., 2018, ApJ, 866, 66

McKernan B., Ford K. E. S., O'Shaugnessy R., Wysocki D., 2020, MNRAS, 494, 1203

Moody M. S. L., Shi J.-M., Stone J. M., 2019, ApJ, 875, 66

Morbidelli A., Szulágyi J., Crida A., Lega E., Bitsch B., Tanigawa T., Kanagawa K., 2014, Icarus, 232, 266

Muñoz D. J., Miranda R., Lai D., 2019, ApJ, 871, 84

Muñoz D. J., Lai D., Kratter K., Mirand a R., 2020, ApJ, 889, 114

Paardekooper S.-J., Mellema G., 2006, A\&A, 459, L17

Paardekooper S. J., Papaloizou J. C. B., 2008, A\&A, 485, 877

Panamarev T., Shukirgaliyev B., Meiron Y., Berczik P., Just A., Spurzem R., Omarov C., Vilkoviskij E., 2018, MNRAS, 476, 4224

Peters P. C., 1964, Phys. Rev., 136, B1224

Ragusa E., Rosotti G., Teyssandier J., Booth R., Clarke C. J., Lodato G., 2018, MNRAS, 474, 4460

Rauch K. P., 1995, MNRAS, 275, 628

Robert C. M. T., Crida A., Lega E., Méheut H., Morbidelli A., 2018, A\&A, 617, A98

Secunda A., Bellovary J., Mac Low M.-M., Ford K. E. S., McKernan B., Leigh N. W. C., Lyra W., Sándor Z., 2019, ApJ, 878, 85

Secunda A., et al., 2020, arXiv e-prints, p. arXiv:2004.11936

Sesana A., Haardt F., Madau P., Volonteri M., 2005, ApJ, 623, 23

Shakura N. I., Sunyaev R. A., 1973, A\&A, 24, 337

Stern D., et al., 2018, ApJ, 864, 27 
Stone N. C., Metzger B. D., Haiman Z., 2017, MNRAS, 464, 946

Syer D., Clarke C. J., 1995, MNRAS, 277, 758

Syer D., Clarke C. J., Rees M. J., 1991, MNRAS, 250, 505

Szulágyi J., Morbidelli A., Crida A., Masset F., 2014, ApJ, 782, 65

Szulágyi J., Masset F., Lega E., Crida A., Morbidelli A., Guillot T., 2016, MNRAS, 460, 2853

Tagawa H., Haiman Z., Kocsis B., 2020a, ApJ, submitted; e-print arXiv:1912.08218,

Tagawa H., Haiman Z., Bartos I., Kocsis B., 2020b, ApJ, 899, 26

Tanaka H., Takeuchi T., Ward W. R., 2002, ApJ, 565, 1257

Tang Y., MacFadyen A., Haiman Z., 2017, MNRAS, 469, 4258

Tanigawa T., Ohtsuki K., Machida M. N., 2012, ApJ, 747, 47

Uribe A. L., Klahr H., Flock M., Henning T., 2011, ApJ, 736, 85

Volonteri M., Sikora M., Lasota J. P., Merloni A., 2013, ApJ, 775, 94

Ward W. R., 1997, Icarus, 126, 261

Will C. M., Yunes N., 2004, Classical and Quantum Gravity, 21, 4367

Yang Y., et al., 2019a, Phys. Rev. Lett., 123, 181101

Yang Y., Bartos I., Haiman Z., Kocsis B., Márka Z., Stone N. C., Márka S., 2019b, ApJ, 876, 122

Yunes N., 2009, GW Notes, 2, 3

Yunes N., Kocsis B., Loeb A., Haiman Z., 2011, Physical Review Letters, 107,171103

Zwick L., Capelo P. R., Bortolas E., Mayer L., Amaro-Seoane P., 2019, arXiv e-prints, p. arXiv: 1911.06024

van der Walt S., Colbert S. C., Varoquaux G., 2011, Computing in Science and Engineering, 13, 22 